\title{
Positron Annihilation and Its Application to Defect Studies in Metals"
}

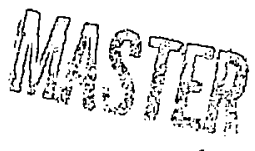

\section{A. N. Goland}

Brookhaven National Laboratory, Upton, New York 11973

\section{INTRODUCTION}

Because the positron was the first antiparticle to be discovered, it is not surprising that the fundamentals of positron physics are by now weIl documented. Modern quantum electrodynamics is capable of providing an explanation for all of the observed properties of the annihtlation process wherein a positron and an electron are replaced in the fimal state by $n$ photons $(n=1,2,3 \cdots)$. In the decade of the fifties, perhaps due to the renewed interest in positrons aroused by quantum electrodynamics, studies of the annihilation process in solids began in earnest. It is not the purpose of this paper to exhaustively review the work which has followed since then. Reviews of this nature are readily available in the open Iiterature. ${ }^{1-6}$ Instead, we will concentrate upon recant developments in the application of positron annihilation to studies of imperfect solids. In particular, we will consider the present status of this field as it applies to metallic solids. After a brief review of positzon physics in section two, in section three we will turn to a description of positron behavior. In perfect metals. Section four will deal with the basic measurements that are performed to extract information about the electronic structure of solids from the annihilation photons. In section five, we will examine the recent experimental results that reveal the connection between positron anmihilation and defects in metals. The sixth section will be devoted to a discussion of the model currently in use to explain the observations described in section five. The last part of the paper will be confined to speculations and suggestions for future work. "Work supported by the U. S. Atomic Energy Commission. 
II. FUNDAMENTALS OF POSTTRON-ANNIHILATION SELECTIOÑ RUÜES

Being conjugate particles, the electron and positron have opposite parity so that the intrinsic parity of the annihilating pair must be odd. Thus the two states ${ }^{1} s_{0}$ and ${ }^{3} s_{1}$ both have odd parity. Now considering the parity of the n-photon states which result from the annithilation process, one finds that when $J=0$ and $n=2$, states of even or. odd parity are allowed. Therefore the ${ }^{{ }} \mathrm{S}_{0}$ state can decay into 2 photons each of energy $\mathrm{mc}^{2}$ (= $\left.511 \mathrm{kev}\right)$. On the other hand, when $\mathrm{J}=1$ annihilation into 2 photons is forbidden. Thus; the ${ }^{3} \mathrm{~s}_{1}$ state cannot decay into 2 photons, but must decay into 3 or more. One-photon anniliflation can occur, but it is essentially a three-bcaly event, and the probability of its occurrence relative to that of two-photon annihilation is $\leq 7 \times 10^{-5}$. Thus, although in principle, one-quantum annihilation could furnish information about electron-electron correlations in solids, in practice it has not become a useful effect.

Experimental measurements of lifetimes in gases lead to the discovery that a positron and an electron can form a bound state. This is positronium, $P_{S}$, which is a hydrogen-like system with an energy level scheme analogous to that of hydrogen, but with a reduced mass of $\mathrm{m} / 2$ instead of $m$ where $m$ is the electron mass. Thus the energy levels of $\mathrm{P}_{\mathrm{S}}$ are given by the relation,

$$
E_{n}=-\frac{m e^{4}}{4 \pi^{2} n^{2}}
$$

The $P_{S}$ ionization potential is $\frac{1}{2} R y=6.8$ eV), and positronfum exists in a ${ }_{S_{0}}$ or ${ }^{3} S_{1} 1 s$ state. The lifetime of the singlet (para) ${ }^{S_{0}}$ state ts $1.25 \times 10^{-10}$ sec. whereas that of the triplet (ortho) ${ }^{3} s_{1}$ state $151.4 \times$ $10^{-7} \mathrm{sec}$. The spin averaged lifetine $=5 \times 10^{-10}$ sec. The ratio of the number of two-photon events to three-photon events is determined by the respective annihilation rates and statistical weights: 


\section{$\frac{\text { 2-photon annihilation rate }}{3(3-\text { photon annihilation rate) }} \equiv \frac{1}{3}$ (1115) $\cong 372$.}

\section{Positron Sources}

A search of the periodic table reveals that there are very few usable positren s.jurces. A usabie positron source in the present context is one that san be produced easily by neutron capture or through a charged-particle reaction, and that has a reasonably long balf-life. A measure of the latter criterion is, of course, the ratic of the half-life to the duration of an experiment. Using these two criteria, one finds that the sources listed in Table $I$ are of experimental value.

Table I. Positron Sources

\begin{tabular}{|c|c|c|c|}
\hline Isotope & Half-1ife & Means of Production & End-point Energy \\
\hline${ }^{22} \mathrm{Na}$ & $2.58 \mathrm{a}$ & ${ }^{25} \mathrm{Mg}(p, \alpha)^{22}$ & $0.54 \mathrm{MeV}$ \\
\hline $65_{\mathrm{Zn}}$ & $245 d$ & ${ }^{64} \mathrm{z}(\mathrm{n}, \gamma)^{65_{\mathrm{Zn}}}$ & $0.33 \mathrm{MeV}$ \\
\hline${ }^{64} \mathrm{Cu}$ & $12.8 \mathrm{~h}$ & ${ }^{63} \mathrm{Cu}(n, \gamma){ }^{64} \mathrm{Cu}$ & $0.66 \mathrm{MeV}$ \\
\hline${ }^{58} \mathrm{Co}$ & $71 \mathrm{~d}$ & ${ }^{58_{\mathrm{Ni}}(\mathrm{n}, \mathrm{p})}{ }^{58} \mathrm{Co},{ }^{\left.65_{\mathrm{Mn}\left(\alpha_{3}, \mathrm{tl}\right.}\right)}{ }^{58} \mathrm{Co}$ & $0.48 \mathrm{MeV}$ \\
\hline${ }^{55} \mathrm{Co}$ & $18.2 \mathrm{~h}$ & ${ }^{58_{\mathrm{Ni}}(\mathrm{p}, \alpha)}{ }^{55} \mathrm{Co},{ }^{56} \mathrm{Fe}(\mathrm{p}, 2 \mathrm{n}){ }^{55} \mathrm{Co}$ & $1.50 \mathrm{MeV}$ \\
\hline${ }^{69} \mathrm{Ge}$ & $40 \mathrm{~h}$ & ${ }^{69} \mathrm{Ga}(\mathrm{d}, 2 \mathrm{2n}){ }^{69} \mathrm{Ge}$ & $1.22 \mathrm{MeV}$ \\
\hline $57 \mathrm{Ni}$ & $36 \mathrm{~h}$ & ${ }^{56} \mathrm{Fe}\left({ }^{3} \mathrm{He}, 2 \mathrm{n}\right){ }^{57} \mathrm{NI}$ & $0.85 \mathrm{MeV}$ \\
\hline $90_{\mathrm{Nb}}$ & $14.7 \mathrm{~h}$ & ${ }^{90} \mathrm{Zr}(\mathrm{p}, \mathrm{n}){ }^{90} \mathrm{Nb},{ }^{90} \mathrm{Zr}(\mathrm{d}, 2 \mathrm{n})^{90} \mathrm{Nb}$. & $.1 .50 \mathrm{MeV}$ \\
\hline
\end{tabular}


Typical source strengths used in experiments range from the order of $\mu C i$ to a few mCi.

The mos: frequently used member of tinis group is ${ }^{22} \mathrm{Na}$. It is readily available albeit expensive in large strengths. Its long half life makes it ideal for all of the typical positron anmihilation experiments. A less expensive alternative is ${ }^{58}$ Co which san be produced in a nuclear reactor.

Recantly, 8 a series of experiments was performed in which each positron source was created in the interior of the metal under investigation. The purpose of this technique was to eliminate possible contributions to measured positron lifietimes arising from source and surface effects. Using photonuclear reactions, Souder was able to produce internal positron sources in Sc, $\mathrm{Zn}, \mathrm{Ni}, \mathrm{K}, \mathrm{Mo}, \mathrm{Ag}$ and $\mathrm{Na}$. While some diffexences were noted in those cross where a comparison could be made, the experimental difficulties encountered tended to introduce new uncertainties. The method, of course, does not lend itself to such widespread usage as the conventional external source technique. Weisberg has studied and discussed the role of surfaces in his thesis. 9 III. BEHAVIOR OF POSITRONS IN METATS

\section{Thermalization}

Note that the end-point energles of the positrons enitted from the sources 1isted in Table $I$ of the preceding section are all of the order of a few tenths of an MeV. As they enter a metal, then, the positrons are moving rapidly, and one must ask how they lose their energy. The relevant questions are: how much time does a position require to reach thermal energies, and how does this time, $\tau_{T}$, compare with the iffetime against annihilation?

These questions were first investigated analyticaliy by Lee-WhitIng. 10 Mis calculation predated the modern many-body techniques and neglected a.1 correlations. Carbotte and Arora 11 re-examined the process using standard propagator formalism. Considering only positron 
election interactions, they assumed that the posftron 1oses energy only through the creation of electron-hole pairs. The rate of energy loss is then given by, the imaginary part of the positron self-energy operator. We note that Carbotte and Arora only calculated the energy-joss rate for low-energy positrons $\left(\mathrm{E}_{\mathbf{t}} \prec 5 \mathrm{eV}\right)$, and thereby neglected plasmon creation In fact, plasmon creation is the dominant mode of energy loss in the kilovolt region.

Carbotte and Arora found that most of the thermalization time occurs toward the end of the positron range. They also noted that the thermalization time increases with increasing electron density. This is because screening increases with electron density, and the stronger the screening the weaker the coupling of the positron to the electronic system.

For positron energies $\leq \sim 0.1 \mathrm{eV}$, they obtained an expression identical to Lee-Whiting's when the Thomas-Fermi screening length for the electron gas is used as the screenjing parameter in Lee-Whiting's formula. Their more exact treatment reveals that at room temperature $\tau_{T}$ for $\mathrm{Na}$ and $\mathrm{Al}$ are much shorter than the 1ifetime for annihilation. In fact this is true for $\mathbb{N a}$ throughout the temperature range from $110 \mathrm{~K}$ to $600 \mathrm{~K}$. However, for $\mathrm{Al}, \tau_{T}>\tau_{\text {Annih. at }} 110 \mathrm{~K}$. Thus thermalization is not complete in this case. On the other hand, Carbotte and Arora pointed out that the time required for a positron to fall to an energy of $\sim 0.1 \mathrm{eV}$ is very short compared to typical lifetimes. Therefore, they assert, it is generaliy a good assumption that the positron momentur is negligible at the instant of annihilation.

Kecently, consideration has been given to effects of the positronphonon interaction in metals by Mikeska. 1.2 He has investigated the role of this interaction in the theralization process as well as its effect upon the positron effective mass. His treatment considers positrons in thermal equilibrium with the electrons and phonons. ye Iinds that although the phonon contribution to the effective mass is 
negligible, for thermal energies the phonon contribution to the momemcurn distribution of the positrons can become much larger than the elecw tron contribution. The rate of energy. loss due to phonon enission at $\mathrm{T}=0 \mathrm{is}$,

$$
R_{p h}(p)_{T=0}=\frac{n \pi}{k_{F}} c^{2} p^{3} \text {, }
$$

where $c$ is the velocity of sound, $k_{F}$ is the wave vector at the Fermi energy, $p$ is the momentum of the positron, and $n$ measures the stirength of the positron-phonon interaction and is of order unity.

The rate of energy loss to electrons in the same region according to Carbotte and Arora is:

$$
\underbrace{}_{\substack{R_{\text {elec. }} \\ \text { hole palrs }}}=\frac{1}{105} \frac{\pi}{k_{F} m^{2} k_{F}} \frac{p^{6}}{.}
$$

Comparing $\mathrm{Eq}, 2$ and $\mathrm{Eq} .3$, Mikeska observed that in the case of sodiun, for example, $R_{p i}>R_{e h}$ for $p^{2} / 2 m<230 K$.

Moreover, he found that the positron momentum distribution is not Boltzmann-1ike. If one identifies the zad moxent of the momeritum distribution with the observed minimum temperature of the positron then for the Mikeska distribution function,

$$
\left\langle\frac{p^{2}}{2 m_{p}^{*}}\right\rangle \approx 1.8\left(\frac{3}{2} k T\right)
$$

Therefore the mean energy of the positron is larger than that predicted on the basis of a Boltzman distribution.

It turns out theoretically that the positron effective inass is nearly equal to unfty, but an apparent effective mass closer to two has been deduced experimentally on the incorrect assumption that the 
positron momentum is describea by a Boltzmann distribution. Thus, Mikeska's results apparently explain most of the discrepancy between the theoretical effective mass derived neglecting positron-phonon interactions, and that deduced from the observed thermal smearing of the experimental monentum distribution

\section{Charge Density Enhancement}

A natuxal quantity to investigate in studying positron annihilation in metals is the annihilation rate, or its reciprocal, the lifetirie. In metals one expects that this quantity will provide informaticn about the electron density at the positron. Generally, in this field, elactron density is described in terms of a dimensionless paraneter $I_{s}$, the radius of the sphere which contains just one electron. Thus, if $\mathrm{n}$ is the number of valence electrons per $\mathrm{cm}^{3}$ then

$$
n=\left[\frac{4}{3} a_{o}^{3} r_{s}^{3}\right]^{-1}
$$

where $a_{0}$ is the radius of the first Bohr orbit.

From Sommerfeld's free electron theory one finds that

$$
\lambda_{\text {Somm. }}=12 \mathrm{x}_{\mathrm{s}}^{-3} \times 10^{9} \mathrm{sec}^{-1}
$$

This result predicts a rather strong variation of lifetime with electron density. However, this dependence is not observed. In fact, the variation of annihilation rate with $r_{s}$ is found to be rather small; most metals exhibit lifetimes of about $1.5-2.0 \times 10^{-10} \mathrm{sec}$. The reason for the failure of the Sommerfeld theory is the strong Coulomb interaction between the electrons and the positron. Thus, it is not the average electron density that is important, but rather the electron density at the positron position. Calculations taking into account this fact have been performed by sevetal authors. ${ }^{13-15}$ 
Most recent is the work of Sjölander and stote ${ }^{15}$ in which a new method for handling screening effects is used to account for the strong electron-electron correlations. The electron distribution is given in terms of the positron-electron static pair-correlation function $g^{+-}(r)$. Figure 1 taken from their paper illustrates the effect of the strong positron-electron attractive potential. The figure clearly shows that a positron in a metal becomes surrounded by its own polarization cloud. The total induced charge is'unity however, so that total charge neutrallty is preserved. The annihilation rates calvulated on the basis of this theory are in good agreement with experiments in aluminum and in the alkali metals, except for cesiun which corresponds to an $r_{s}$ of about 5.7. The results become unreaistic beyond an $r_{s}$ of about 5 . At present, there is no reason to believe that positroulun is formed in metals. If it were, a so-far unobserved long iffetime should arise from ${ }^{3} \mathrm{~S}_{1}$ annihilation. Or else, a rapid triplet-singlet conversion could give rise to an unobserved lifetine equal to four times the ${ }^{1} \mathrm{~S}_{0}$ lifetime, i.e., $5 \times 10^{-10} \mathrm{sec}$. The primary reason qiven for the absence of positronium is that a posttrontum-1ike bounc state cannot form at normal metallic electron densities, but only at densittes such that $r_{s} \geq 8.6 .^{16}$ For real metals $1.8 \leq r_{3} \leqslant 5.5$.

IV. EXPERIMENTAL METHODS OF INVESTIGATING POSTTRON

\section{ANNTHILATION IN SOḶIDS}

\section{General Remarks}

Since the two antiparticles disappear during an encounter, all information about the event is carried away by the photons. We have noted earlier that two-quantum annihflation is most common, and it is the event mos: often studiea in metals.

Basically there are three types of measurements commonly performed. 
One is a measurement of the positron lifetime, another is the Dopplet broadening of the annihilation line, and the third is a measurement of the angular-correlation of two-photon annihilation radiation.

Suppose that $\Gamma^{2}(p) d p$ is the probability per unit tine that annihilation into two photons whose total momentum lies batween $\mathrm{p}$ and ptdp takes place. Then it has been shown ${ }^{17}$ that if the electron is in a state $\psi_{i}$, and the positron in state $\psi_{t}$,

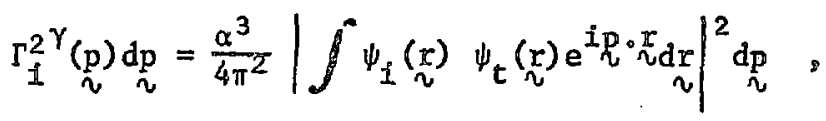

where atomic units have been used and the atomic unit of time is $2.42 \times 10^{-17} \mathrm{sec}$. The annihilation rate into any total momentum then must be,

$$
\Gamma_{i}^{2 \gamma}=\int \Gamma_{i}^{2}(p) d p=\frac{\alpha^{3}}{4 \pi^{2}} \iint \psi_{i}(r) \psi_{t}(r) e^{i p} \cdot \underset{v}{r} d r \mid d p .
$$

By Parseval's theorem this becomes

$$
\Gamma_{i}^{2 \gamma}=2 \pi \alpha^{3} \int\left|\psi_{i}\left(r_{n}\right) \psi_{t}(r)\right|^{2} \mathrm{dr}_{n}=2 \pi \alpha^{3} \int\left|\psi_{i}(\underline{r})\right|^{2}\left|\psi_{t}(\underline{r})\right|^{2} \mathrm{~d} r
$$

the partial annihllation rate, $\lambda_{i} \cdot$. Summing over all occupied electron states we obtain the total annihilation rate, the reciprocal of which is the lifetime of the positron:

$$
\tau^{-1}=\sum_{\text {occe } i} r_{i}^{2 \gamma}
$$

\section{Angular-Correlatic}

Now let us constder a typlcal angular-correlation experiment. The sample and positron source are located, as shown in Figure 2, midway 
between a pair of photon detectors. Generally a linear sitt is placed in front of each detector and the sample-detector distance is made long Enough so that the angular resolution is between 0.5 and 1.0 mrad. Appropriate lead shielding is placed around the source so that the detectors see only photons created in the sample.

If the anthilating pair were at rest in the center-of-mass systen, then the two quanta would be enitted preciseiy in opposite directions in the laboratory system. We have concluded in our earlier discussion of thermalization that for most purposes one may assume that the positron is thermalized and, hence, it is essentially at rest." The annihilating electron, however, is not. Therefore, the center of mass of the anihilating pair is in motion, and the two photon directions deviate slightly from $180^{\circ}$. relative to one another in the laboratory system. This slight deviation is what gives rise to the demand for high angular resolution. In practice one arm of the instrument shown in Figure 2 is held stationary while the other is moved in small angular increments in the $z$ airection, for example. In order to obtain sufficient serisitivity with the avallable positron sources one employs largevolune detectors whose long dimension is paralle1 to the collimating s1its. Thus, the detectors are insensit ve to the $x$ and $y$ components of momentum, and the experiment determines only one component, say $\mathrm{P}_{z}$. From the figure we see that to a good approximation for the smail angular range under consideration (roughly $0-50$ mrad.) $P_{2}=$ mce ( $=c \theta$ in atomic units). In terms of the expressions giver in the previous section the rate of annihilation into the momentum region between $P_{z}=c \theta$ and $c(\theta+d \theta)$ becomes, 18

$$
\mathbb{N}_{z}(\theta)=\sum_{I} c \int_{-\infty}^{\infty} \int_{-\infty}^{\infty} \Gamma_{1}\left(p_{x}, p_{y}, c \theta\right) d p_{x} d p_{y},
$$


and the area under the angular correlation curve is,

$$
\int_{i}^{\infty} N_{z}(\theta) d \theta=\sum_{i} \int_{\infty} \int_{i}^{\infty} \Gamma_{i}\left(p_{x}, p_{y}, c \theta\right) d p_{x} d p_{y} d \theta
$$

a quantity proportional to the total annihilation rate.

of course, in practice, the area under the actual measured angularcorrelation/curve is just equal to the total number of coincidence counts collected. But if the nomalization factor were known this area sond be converted into an amihilation rate or a total lifetime.

If one calculaces electron wave functions for the electrons in various shells of an atom or for valence electrons, and if one also knovg the positron vavefunction, then it is possible to generate a

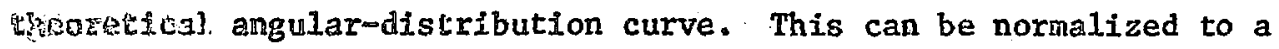
thessured distribution, and one then can make some assessment of the retcing emtribution which electrons in different states make to the observed annihilation process. An example of the method applied to

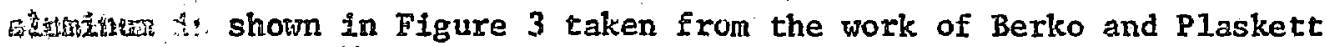
(Fits. 12, Nei. 18). The theoretical curves, shown as solid lines, Heve dears tormalized to the experimental counting rate at $0^{\circ}$. The open wreles the the experimental points. The parabolic curve corresponds co cfe dular distribution arising from anishilation with the three valeme lectrons, while the other curve represents the contribution frouth L-sh:tl electrons. This illustrates the high-momentum contribution the angular distribution which comes from core annihflations. Cons ldering annihilation in a gas of free electrons, it is easy to ses hort the parabolic valence-electron distribution in Figure 3 comes atsats. Whow that the momentum of the photons comes from the electxope sin the positron is essentially at rest. The electron momenta are distributed uniformly throughout a sphere whnse radius is the Farmi moskntum. The conventional method of measuring an angular dis . kribution corresponds to taking a slice through the sphere at a 
transverse momentum $k_{z}=m c \theta$. The area of this silce as shown in Figure 4 is going to be proportional to the rate of annihilation into the angular region $\theta$ to $\theta+d \theta$. But this area is proportional to $k_{F}^{2}-k_{z}^{2}$, and so the expected distribution for a Fermi gas is an inverted parabola. Many metals exhibit this form of low-momentum distribution (aluminum, for example in Tgure 3). Consequently one usually speaks of this portion of an angular-distribution curve as the valence or conduction-electron part. We shall Tearn shortly that it has become common practice to resolve a measured distribution into a parabolic and a Gaussian part for the purposes of analysis. This is an approximation that is valid for some solids but it assumes, among other things, that the valence electrons make no contribution to the high-momentum tail of the angularocorrelation distribution.

\section{Doppler Broadening}

We noted in the preceding section that the detectors used In angular-correlation experiments are insensitive to the component of electron momentum 1ying along the direction of the emitted photons. This component gives rise to the usual Doppler broadening of the annihilation photon at $511 \mathrm{keV}$. If $\mathrm{E}_{0}$ is the rest mass of the electron, then the broadening $\Delta E$ is given by the relation

$$
\Delta \mathrm{E} / \mathrm{E}_{\mathrm{O}}=\left(\mathrm{v}_{\mathrm{com}}\right)_{\mathrm{x} / \mathrm{c}}
$$

Assuining that the positron is at rest, we find that the veloctty of the center of mass, $v_{\mathrm{c}}$. equals $1 / 2 \mathrm{~V}$, the electron velocity. Thus,

$$
\Delta E / E_{0}=\frac{1}{2}\left(v_{e}\right)_{x} / c
$$

or

$$
\Delta E=\left(p_{x} / 2 m a\right) E_{0}, \text { where } p_{x} \text { has } 1 \text { ts usual meaning. }
$$


If the medium is isotropic with respect to the annihilation prom cess, then all momentum components are equivalent. This means that $\mathrm{p}_{\mathrm{x}}$ is equivalent to $p_{z}=m_{0} c \theta$ as determined in the usual angular-correlation experiment.

Ihus,

$$
\Delta \mathrm{E}=\left(\mathrm{m}_{0} c \theta / 2 \mathrm{~m}_{0} \mathrm{c}\right) \mathrm{E}_{0}=\frac{1}{2} \mathrm{E}_{0} \theta=255.5 \dot{\theta e V}
$$

where $\theta$ is in milliradians and $\triangle E$ in electron volts. So we see that a one milliradian angular resolution corresponds to a Doppler broadening of $255.5 \mathrm{eV}$, and in principle, the two experimental techniques can furnish the same information. If the instrumental resolution is unFolded from the Doppler-broadened annihilation peak, then the electron momentum distxibution will be recovered.

The method of performing these experiments is to use a Ge(Li) detector as part of an energy spectrometer. The instrumental resolution function $f\left(E, E^{\prime}\right)$, is determined by least-squares fitting of a multiparameter analytical expression to the shape of an unbroadened line from a known decay scheme at an energy conveniently near 511 kev. If the true energy distribution in the Doppler-broadened line is $N(E)$ then the convolution function

$$
g(E)=\int \mathrm{N}(E) f\left(E, E^{r}\right) d E^{\prime}
$$

can be calculated and compared with the experimental data point by point using least-squares analysis. This procedure yields the true distribution $N(E)$. Most of the difficulty in obtaining a good fit artses from the determination of the resolution function. Nevertheless, the unfolded angular distributions agree well with angular-correlation data as can be seen in Fig. 5 taken from the work of Rama Reddy and Carrigan on copper and zinc. " They have prepared a table in which a 
comparison of the two techniques is made. That table is reproduced here as Table II on the following page.

The limiting factor in these experiments at present is the FWIM of the Ge(LI) detector. The best available detector has a FWHM of about $1.1 \mathrm{keV}$; this corresponds to an angular resolution of about 4 mrad, not very good by comparison with angular-correlation instruments. The FWHM of these detectors is given by,

$$
\text { FWHM }=2.36 \sigma(E F \varepsilon)^{1 / 2}
$$

where $\sigma$ is the statistical width, $B$ the energy of the photon, $\varepsilon$ the energy required to produce a charge pair, and $F$ is a characteristic of the charge-paix creation process called the Fano factor. It is a measure of the fraction of the total number of electron-hole pairs created that is statistically independent. If the FWHM were smaller then there would be real promise for this method. We shall see in a later section that Mackenzie and his colleagues are even now using Doppler broadening successfully to study imperfect metals and alloys. No doubt others will follow their lead in the near future.

The method has one distinct advantage. It requires only one detector and no coincidence circuitry. Data can be collected rapidly and the statistics are, therefore, very good. Stabilized electronics are essential, however.

\section{Lifetimes}

The measiured lifetimes of positrons in metals are all around 0.2 nsec. There are now commercially available electronic components that can be assembled to measure such short times. They operate near the limit of their capability, however, and great care is required to achieve reproducible results. 
Table II. Comparison of 2-Photon Angular Correlation and DopplerBroadening Techniques (Ref. 7).

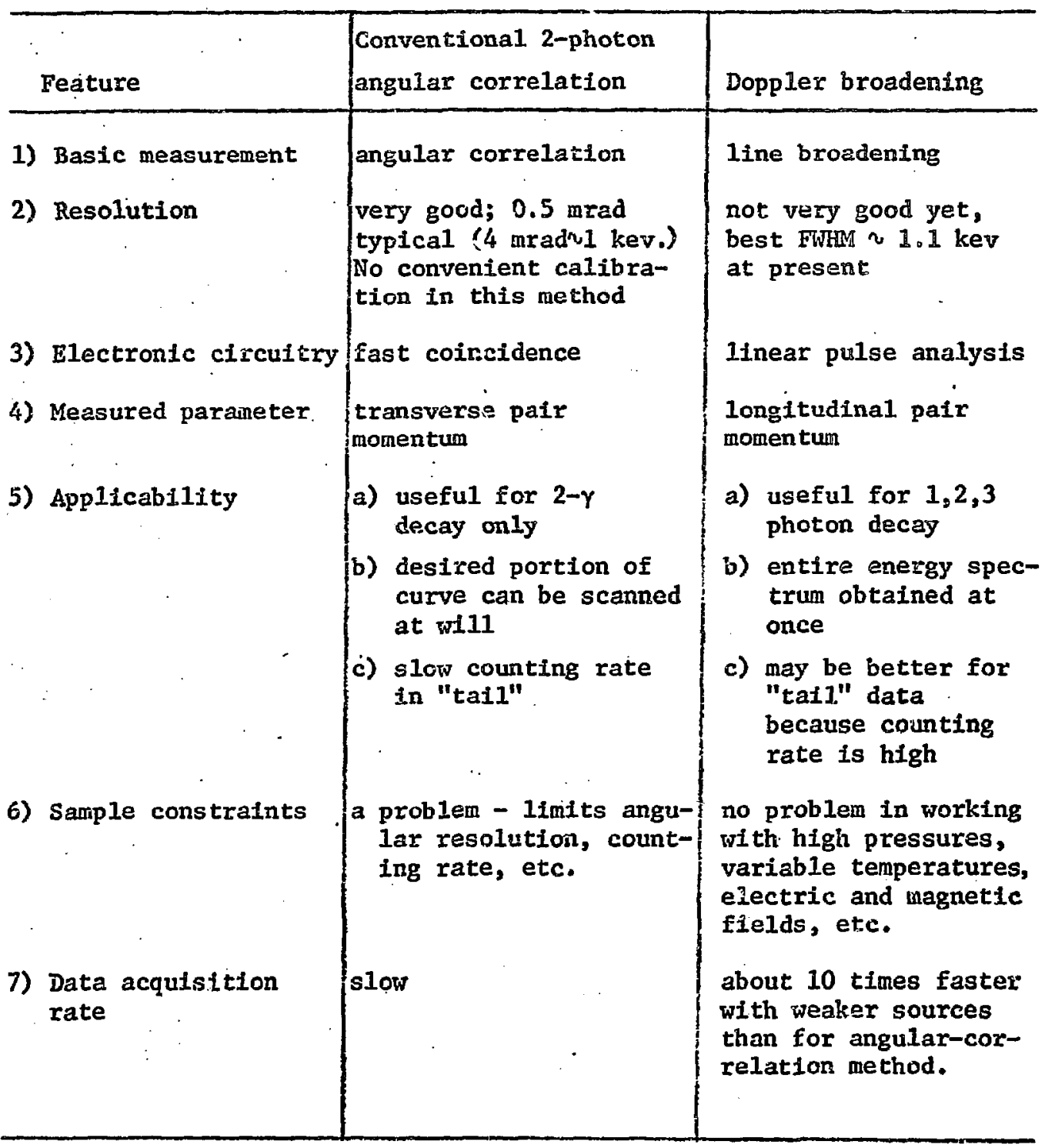


The standard method of measuring 11fetimes employs delayed-coincidence counting, and a typical system is shown in the block diagram of Fig. 6. The system includes a time-to-amplitude converter by means of which the elapsed time between the emission of the positron and its annihilation in the sample is converted to a proportional pulse which is stored in a multichannel analyzer. The distribution of time intervals, and therefore pulse heights, is an exponential whose decay time is the mean life, $\tau$, provided that the natural delay is 1ong compared to the width of the prompt resolution curve. The time resolution is determined by measuring the pulse-height distribution from a source that emits two coincident gamma rays, for example ${ }^{60} \mathrm{Co}$. The typical spectra would appear as. In Fig. 7. The Iffeitme equals the difference in the centroids of the two curves shown in Fig. 7. Newton has showit that it also can be determined from the slope of the tail of the delayed coincidence spectrum. However, generally the analysis is performed by computer fitting of a sum of exponentials to that part of the delayed curve corresponding to large positfve delays. The prompt resolution curve is approximated by a Gaussian and folded with the exponentials in a least-squares calculation. In the case of metals, it is generally assumed that the delayed curve consists of only two exponentials. One exponential correspoxids to the desired lifetine while the other is of weak intensity (i $2-5 \%$ ) and artses from annihilation within the source. The latter usually ylelds a lifetime of $\approx 0.5$ nsec. In the next section we shall learn that it is of interest to look for two real lifetimes that are close to one another in metals. However, successful attempts to resolve ther have not been reported to date.

The use of delayed-coincidence circuitry requires that care be taken to achleve good electronic stability. The major problem arises from the so-called "walk" effect in fast-pulse spectrometry. Walk arises because puises of the same rise time but different amplitude cross the zero-level discriminator at slightly different times. Thus, 
the timing has an inherent error in it. Methods have been devised for reducing this effect and are now in common use. The stability of the electronic system is both temperature and humidity sensitive. It is essential that the experiments be conducted in an area where good control over these ambient conditions exists. Recent. developments in integrated circuitry may reduce the problem, for small IC chips lend themselves to temperature regulation through the use of cooling devices such as thermoelectric coolers.

y. POSITRON ÄNIHILATION IN IMPERFECT METALS - EXPERTMENT

\section{Defects in Metals}

A perfect metal is an idealization that is not realized in nature, although in sone special cases it can be closely approximated. Nevertheless, many properties of real metals are determined by their defect structure. The defects may take the form of vacant lattice sites, interstitial atoms of the host or of an impurity, dislocation netwo:ks, grain boundaries, or foreign precipltates, for example.

In recent years, it has been discovered that positrons are extremely.sensitive to the presence of certain kinds of lattice imperfections. Because an understanding of defects in solids is so vital to an understanding of the behavior of solids, the prospect of a new probe for studying defects has excited great interest. In sections V-VI we shall examine these recent developments, discussing first the experimental observations, and second the models that have been proposed to explain the data. The field is in its infancy, and in the next fey years we 111 determine the extent of its usefulness. Already there are those who believe that positron annihilation offers the best hope for measuring vacancy formation energles accurately. 
We need not discuss defects themselves in detall for the purposes of our discussion. The literature covering this subject is extensive and readily available. ${ }^{19-21}$. Moreover, in confining our discussion to positron-defect interactions we are aware that we are omitting a large body of experimental results dealing with the behavior of positrons in metals. In fact, the effects to be described have a direct bearing upon these other studies. For it quickly will become apparent that the results of angular-correlation or lifetime measurements are affected by the state of the sample as regards deformation and temperature, to mention two important parameters. Thus, the interpretation of data must take into.account the history of a sample as well as the conditions that applied during its use in an experiment.

By now a number of Investigators have confirmed the role of defects in positron annihilation and we need only summarize some of the results in this paper. Several years ago, Dekhtyar ${ }^{22}$ published a review in which he described many experimental observations of positron annihilation in metals and alloys containing lattice defects. For a more complete review of experimental data one may refer to his paper.

\section{Experimental Results}

Piastle Deformation. It was probably Dekhtyar and his colleagues 23 who first noted that plastic deformation measureably altered the shape of a two-photon angular-correlation curve. Their Investigation began with Iron and Iron-nickel alloys, but it subsequently extended to a number of cubic metals and alloys. In every case they observed that after deforming the samples by rolling to $70-80 \%$ reduction two changes took place:

1) The maximum counting rate increased, and

2) The BWHM decreased. 
Taken together these two changes were interpreted as being due to a decrease in positron interactions with core electrons and an increase in their interaction with conduction electrons. This might arise from some redistribution of electrons in the $s$ and $a$ bands due to the strain introduced by deformation.

Several years elapsed before Berko and Erskine 24 investigated the 2-quantum angular correlation in plastically-deformed aluminum, and corfirmed Dekhtyar's observations. Their aluminum samples had a purity of 99.99\% and were $0.1 \mathrm{in}$. thick. After undergoing an annealing treatment at $600^{\circ} \mathrm{C}$ for 3 hours and a slow cooling to room temperature the samples were hammered to reduce their thickness from 10-16\%. These samples exhihited a narrowing for small values of $\theta$ and a smearing at $\theta_{F}$ (the angle corresponding to the Fermi cutoff) relative to the antealed state. Reannealing produced complete overlap of the data with those from the original annealed state of the sample.

To eliminate possible surface effects Berk and Erskine used two positron sources with different end-point energies: ${ }^{22} \mathrm{Na}, 59 . \mathrm{kev}$, and ${ }^{69} \mathrm{Ge}, 1.22 \mathrm{MeV}$. The results were indistinguishable thus proving that the effect was associated with the volume and not the surface of the sample.

The results of these experiments suggested that positrons annihilate preferentially in regtons of lowered electron densicy near defects. Berleo and Erskine pointed out that the positrons could be preferentially attracted toward the dislocations produced by deformation and might even become bound. This idea appears plausible in view of the earlier calculation of Cottre11, Hunter and Nabarro ${ }^{25}$ who showed that the strain field around an edge dislocation induces a charge redistribucion and a consequent electric dipole at the dislocation. The potential due to a dilacation $\Delta$ was found to be $v^{n / 4 / 15}\left(\hbar^{2} k_{F}{ }^{2} / 2 m\right) \Delta$. This is the electrical interaction energy of a unit charged particle placed in any part of the strain field. If the positron wavefunction peaks in the vicinity of the 
dipole, or if, indeed, the increased kinetic energy due to localization is outweighed by the interaction energy, thereby leading to a bound state, then this effect might help to explain the changes observed. If: does not, however, suggest why fewer annihilations with high-momenta electrons should occur in the vicinity of the dislocation.

Berko and Erskine also pointed out that vacancles are produced during plastic deformation, and could serve as positron traps. They suggested that if this were so then the same defect might explain tha increased Iifetimes observed by Mckee et al. ${ }^{26}$ in deformed aluminum, and the change in angular correlation with temperature ubserved earlier by Mackenzie et $2{ }^{2}{ }^{27}$ for example.

Dekhtyar et ai. 28 also reported the first study in single crystalls, using deforined molybdenum crystals with (110) or (100) faces. They cold rolled the spe:amens to produce a deformation of $17 \%$ and then annealed them after each experiment, first to $1000^{\circ} \mathrm{C}$, then to $1500^{\circ} \mathrm{C}$ and finally to $1800^{\circ} \mathrm{C}$. When positrons were incident on the (110) face the 2-plioton angular-correlation curve exhibited the expected narrowing and increase in peak height (by $\sim 20 \%$ ). By decomposing the distribution into a parabola plus a Gaussian, Dekhtyar et al. were able to define the relative probability of annihilation with conduction electrons as the watio of the area under the parabola to the total area. They found the surprising result that the ratio for $17 \%$ deformation in the single crystal seemed to be equivalent to that caused by $80 \%$ deformatlon of polycrystals. Also the changes were much larger in the (110) than in the (100) sanple. Annealing caused the distribution curves to return to the shape arising from the initial undeformed crystal. Delkhtyar and his colleagues suggest that one should investigate changes In the shape of the Fermi surface in deformed crystals. These experiments and others similar to them confirmed the relationship between plastic deformation and angular-correlation data. Similar and related 
effects were observed at about the same time in 1ifetime and Dopplerbroadening experiments.

For example, Grosskreutz and Millett ${ }^{29}$ introduced dislocations and vacancies into copper and aluminum by means of cyclic deformation. The 4-9 aluminum was cycled 8000 times at room temperature while 2-9 copper sustained 3000 deformation cycies at the same temperature. Measurements at room temperature showed the following changes in mean lifetime:

\begin{tabular}{|c|c|c|c|}
\hline \multirow{2}{*}{$\frac{\text { Metal }}{\text { A1 }}$} & \multicolumn{2}{|c|}{ Treatment } & \multirow{2}{*}{$\frac{\tau}{204 \mathrm{psec}}$} \\
\hline & 1) & annealed & \\
\hline & 2) & $\begin{array}{l}\text { fatigued ( } 8000 \\
\text { cycles) }\end{array}$ & $258 \mathrm{psec}$ \\
\hline \multirow[t]{3}{*}{$\mathrm{Cu}$} & 1) & annealed & 177 psec \\
\hline & 2) & $\begin{array}{l}\text { fatigued ( } 3000 \\
\text { cycles) }\end{array}$ & 259 psec \\
\hline & 3) & $\begin{array}{l}\text { fatigued and } \\
\text { annealed for } \\
15 \text { min } 375^{\circ} \mathrm{C}\end{array}$ & 259 psec \\
\hline
\end{tabular}

It is known that the annealing treatment at $375^{\circ} \mathrm{C}$ dor not remove the equilibrium dislocation distribution in fatigued copper although it will remove prismatic loops and perhaps vacancies. Grosskreutz and Millett thus concluded that the observed enhancement in iffetime had its origin in the dislocation network, but that the specific morphology of the network was unimportant, at least in copper. They suggested that one should observe two lifetimes in deformed metals if they can be resolved.

Doppler-broadening experimerits also have been directed toward examining the behavior of positrons in imperfect metals. Using a $\mathrm{Ge}(\mathrm{Li})$ spectrometer with a resolution of $1.2 \mathrm{keV}$ at $514 \mathrm{keV}$, Mackenzie was able to distinguish differences in lineshapes in different metals and changes in lineshape for a given metal under different strains. He cold-rolled samples of aluminum, gold and copper to double their 
intial area and found definite changes in Ifneshapes In each case the line broadening diminished, the largest effect beting observed in copper. On the other hand, indium exhibited no naxroving following defortarion but a marised narrowing with increasing temperature. We will say more about the temperature effect siortly. Since the Doptler broaciting is only senstelve to changes in the core contribution to the momentum distribution. Mackenzie concluded that the line narrowing must arise from trapping of positrons at vacancies and dislocations where they annihilate more fiequentily with low-momenten electrons than they do in the perfect metal. Mackenzie and his colleagues 31 extended their investigations to high-purity copper and to an aluminum ailoy known as 6061. They defined a lineshape parameter,

$$
S=\frac{\text { Ccounts in fixed no of channels in the center of the peak }}{\text { Area of the peak }}
$$

The numerator was chosen so that $s \varkappa 0.5$. An interesting point nored by these investigators is that the good statistical properties of Doppler-broadening measurements compensate for the small changes in S usually observed. For example, S changed by only $3.5 \%$ between the annealed and the severely cold-worked spectimens. However, because the quantity $S$ follows a binomial distribution rather than the usual Gaussian distribution, the error for $5 \times 10^{5}$ counts in the peak was only $0.35 \%$. The resolution of the spectrometer was $1.6 \mathrm{keV}$ for the $514 \mathrm{keV}$ line of ${ }^{85} \mathrm{sr}$. On the assumption that the value of $\mathrm{s}$ is linearly related to the fraction of trapped fosityons, $n_{t}$, Mackenzie et al. found that,

$$
n_{t}=A+B \log D \text { for } 20 \%<n_{t}<95 \% \text {, }
$$

where $\mathbb{D}$ is the percent deformation. They we also able to measure the surface hardness of their specimein, and found that it correlated 
very sell with the values of $\eta_{t}$ derived from lineshape changes for $D<n_{n} 34 \%$ ( $F I g$. 8). Thus, these data heid out the promise of determinir dislocation densitfes from Doppler-broadening. However, the Euthy:s pointed out, correctly, that a quantitative link depends upon thessy of posteron-defect interactions, and that the predictions of such theory are sensitive to the form of the interaction chosen. Warits groups have used different models of the positron wavefunction, steasilug the positron as a plane wave, a diffusing wave packet or a clestical particle in thermal equilibrium with the sample. Each nodel can sead to a different interpraciation of the point-defect density presant.

Femperature Pfects. Perhaps the first detailed examination of the semperature dependence of positron annihilation in metals was that contrited by decleazie and his collaborators. 27 They found that Inchin, cadoium, and zine exhibited markedly narrower angular-correlation discributions at elevated temperatures. An increase in peak

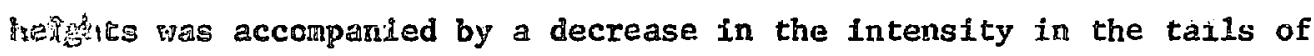
distributions. Some of the metals investigated did not respond in the sape narner, however. For example, lead, tin, aluminum and bismuth shatus.d orl small variations with temperature. These colid be understan in terms of the lattice thermal expansion. At the time, the raf of defectis pas not fully appreciated. Nevertheless, these authors the solnt out that certain metals evidently exhibit some of the disfircin properties of their liquid state before reaching their melcing trition.

Sonewhat lacer, Macilenzte and his associates ${ }^{32}$ reeramined the Rersererure effect using mean lifetimes as an indicator racher than arg lar discributions. They obsained data from cadmiun, indium, atnc。 allin inus and copper. With the exception of copper, whose temperature Fis

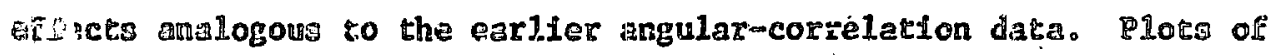


the annihilation rates versus temperature exhibited shapes that were similar to plots of the tail intensity versus temperature in the angulat-correlation tork. As usual, a weak second component of lifetime (2-3\%) was observed, and was attributed to surface effects following Weisberg and Berko. ${ }^{33}$ The intensity of the weak component was determined at room temperature and at high temperature, and the average value was used to correct data at intermediate temperatures. Because of the absence of any hysteresis in their data Mackenzie et a1. concluded that the effect they observed could not be attributed to dislocations. In general, the lifetimes increased with increasing temperature, and tended to reach a plateau at some elevated temperature. The only physical change that Mackenzie et al. could link with the observed. effect was the variation in the equilibrium vacancy concentration with temperature. They, therefore, came to the conclusion that a positronvacancy interaction must exist, and that the lower electron density expected in the vicinity of a vacancy must account for the longer lifetimes associated with positrons in these defects. They did not, however, anticipate that vacancies actually would form bound states at vacant lattice sites.

Shortly thereafter, Brandt and Waung ${ }^{34}$ demonstrated that sodiun and gallium showed large changes in their major lifetime component upon melting. These authors pointed out that the prediction of the freeelectron theory for electron-density changes on melting are much too sma11 to account for their results. They, too, suggested that observed changes were due to annihilation at vacancies with mostly low-nomenta electrons (as required by angular-correlation data on liquid metals). Brandt and Waung pointed out that if vacancies were responstble for what they observed then the lifetime change would be positive if the vacancy concentration, $C_{V}$, were such that 


$$
C_{V}=\left(\frac{\Delta V}{3 V}-\frac{\Delta a}{a}\right)>0 \text { in the Iiquid. }
$$

$V$ represeniting the sample volume, and $a$, the lattice parameter. In gailium, $\Delta V / 3 V<0$ so that $\Delta a / a$ must be even more negative to account for the observed increases in lifetime. According to Brandt and Waung this requirement is consistent with $\mathrm{x}$-ray data that suggest clustering in liquid gallium. Such a phenonenon could yield a negative $\Delta a / a$ and would diminish at Increasiag temperatures in the melt. This, in turn, would cause the lifetime increment to decrease as was observed at temperatures well above the melting point.

All of the foregoing experimental observations seemed qualjtatively consistent with the concept of a strong positron-vacancy interaction. As early as 1965 , Brandt ${ }^{35}$ had proposed a simple rate theory to explain a related phenomenon. This model subsequently was applied to metals in more explicit terms by several groups. It has become known as the "trapping model".

\section{THE TRAPPING MODEL}

Bergerson and Stott, and Connors and West in similar treatments described a phenomenological model of positron annihilation in metals, assuming that vacancies act as positron traps.

Suppose that there are two annihilation rates associated with a meta $1,-\lambda_{1}$, corresponding to the decay rate in the perfect metal and$\lambda_{2}$ corresponding to the positron decay rate in vacancies. Suppose also, that the rate at which positrons are trapped at vacancies is given by the relation

$$
K=\mu c(T),
$$

where $\mu$ is presumed to be a constant of the material and $c(I)$ is the 
vacancy concentration at temperature $T$. Then the rate equations describing the time dependence of the number of free and trapped positions are, respectively,

$$
\begin{aligned}
& \dot{\mathrm{n}}_{1}=-\lambda_{1} \mathrm{n}_{1}-\mu \mathrm{cn_{1 }}, \text { and } \\
& \dot{\mathrm{n}}_{2}=-\lambda_{2} \mathrm{n}_{2}+\mu \mathrm{cn}_{1},
\end{aligned}
$$

where, inftially, $n_{1}+n_{2}$ equals $N$, and $n_{2}(0)=0$. Solving these equations one obtains for the rate of annihilation $R(t)$ at time $\tau$, the expression,

$$
\begin{aligned}
R(t) & =-N^{-1} \frac{d}{d t}\left(\mathbf{n}_{1}+\mathbf{n}_{2}\right) \\
& =\frac{\lambda_{2} \mu c \exp \left(-\lambda_{2} t\right)+\left(\lambda_{1}-\lambda_{2}\right)\left(\lambda_{1}+\mu c\right) \exp \left[\left(-\lambda_{1}-\mu c\right) t\right]}{\lambda_{1}+\mu c-\lambda_{2}} .
\end{aligned}
$$

At time $t=0$,

$$
\begin{aligned}
R(0) & =\frac{\lambda_{2} \mu \operatorname{ct}\left(\lambda_{1}-\lambda_{2}\right)\left(\lambda_{1}+\mu c\right)}{\lambda_{1}-\lambda_{2}+\mu c}=\frac{\lambda_{1}{ }^{2}-\lambda_{1} \lambda_{2}+\lambda_{1} \mu c}{\lambda_{1}-\lambda_{2}+\mu c} \\
& =\lambda_{1} \frac{\left(\lambda_{1}-\lambda_{2}+\mu c\right)}{\lambda_{1}-\lambda_{2}+\mu c}=\lambda_{1} \text {, the 'Eree" decay rate. }
\end{aligned}
$$

The mean Iifetime predicted by this model turns out to be

$$
\bar{\tau}=\lambda_{2}^{-1}\left(\lambda_{2}+\mu c\right) /\left(\lambda_{1}+\mu c\right) \text {. }
$$


The mean Iffe consists of contributions from the two decay modes which are characterized by the lifetimes $\lambda_{2}^{-1}$ and $\left(\lambda_{1}+\mu \mathrm{c}\right)^{-1}$. Bergersen and Stoth ${ }^{36}$ pointed put that these two decay modes make equal contributions whem

$$
\mu c \approx \lambda_{1}-\lambda_{2}
$$

Now the vacancy concentration at any temperature $T$ is given by a Boltzmann expression, viz.,

$$
C: T)=\operatorname{Bexp}\left(-E_{I V}^{F} / k_{B} T\right)
$$

where $B$ is a factor arising from the configurational entropy of the vacancy in a particular lattice structure, $k_{B}$ is Boltzmann's constant, and $E_{F}$ is the formation energy of the vacancy. If $\lambda_{1}$ and $\lambda_{2}$ are known from low- and high-temperature data, respectively, then the experimental temperature dependence of $\bar{\tau}$ can be used to determine the "critical" temperature at which

$$
\bar{\tau}=\frac{\lambda_{1}}{\lambda_{2}\left(2 \lambda_{1}-\lambda_{2}\right)},\left(\mu c \approx \lambda_{1}-\lambda_{2}\right) .
$$

Inowing this temperature, Bergersen and Stott evaluated the assoefated "criticai" vacancy concentration by using assuned values for the vacancy formation energy, $\mathrm{E}_{\mathrm{IV}}$. They allored $\mathrm{B}$ to be unity whereas it is often $\approx 10$, and they used somewhat arbitrary values of $E_{I y}^{E}$. Nevertheless, even if the correct values of $B$ and $\mathbb{F}_{I V}^{F}$ are used In the expression for $c$, one stlll arrives at their conclusion that positrons are very sensitive to the presence of vacancies im fractional concentrations as.$\rightarrow$ as $10^{-6}$. This suggests that positrons offer an opportunity to study equilibrium concentrations of vacanctes in metals. 
Using the same model, Connors and west ${ }^{37}$ have drawn equivalent conclusions. They proposed, following earlier work ${ }^{3}$, that the constant II can be equated to $\sigma_{c} \vec{V}$ where $\sigma_{c}$ is the capture cross section of the vacancy and $\bar{v}$, the thermal velocity of the positron $\left(\alpha T^{1 / 2}\right)$ 。

Hautojärvi et al. 38 applied the trapping model to the measurement of positron lifetimes in deformed aluminum. They reasoned that since deformation produces vacancies and dislocations, the trapping model should be applicable. Their paper is especlally valuable because it illustrates the dffficulties encountered in analyzing data on lifetimes In terms of two competing decay modes. Hautojärvi et al. found that attempts to fit their data with two exponentials plus a source term failed. In fact after subtracting a source term of about $2 \%$, and using a one-exponential fit they still found that the variance had reason-. able values only for the undeformed and highly-deformed specimens. They concluded that these results again verified that only one 1ifetime exists at each extreme, and that intermediate lifetimes are a mixture of these cwo states. Recognizing that $k$ is the only paramster In the trappins model snce $\lambda_{1}$ and $\lambda_{2}$ are determined, they followed a fitting procedure to obtain values of $k$ as a function of deformation from the experimenta: annitilation rates. The values of the variance were reasonable, but not as good as they had anticipated.

Since vacancies make a negliglble contribution to the positron lifetime in aluninum at $300 \mathrm{~K}$ where che data were taken, Hautojärvi and his colleagues concluded that their observations were affected only by dislocations. However, their attempts to relate the trapping rate, $x$, to the dislocation density by electron microscopy were not especially noteworthy.

of course, similar attempts have been made to explain angularcorrelation data on the basis of the trapping inodel. These include the work of Dekhtyar on deformed metals, 22,28 connors et a1. 39,40 on quenched cadniun and Kugmiss et a $1^{41}$ on electron-1rradiated platinur. 
In all cases, the changes in the angular-correlation distributions were the sane as those arising from increases in sample temperature. Most of the results have been published in brief form. However, two conplete papers have appeared recently. In the first, Snead et al. 42 discussed the influence of defects on the angular-correlation distribution in electron-irradiated and in deformed iron. In the second, Connors et al. ${ }^{40}$ considered the effects of vacancies on posicron annithilation in cadmium.

Snead et al. have shown that one can deduce trapping cross sections from a combination of angular-correlation and lifetime data by using the trapping model. They have shown that if the angular correlation curve can be described by a combination of a parabolic and a Gaussian distribution, then the change in the peak height of the total curve can be expressed as $\checkmark$

$$
\Delta H=(\Delta H)_{s} n_{t}
$$

where $(\Delta H)_{S}$ is the difference in the peak heights for saturation and for "zero" vacancy concentrations, and $n_{t}$ is the fraction of trapped positrons. The trapping model in turn requires that

$$
\eta_{t}=\frac{\mu c}{\lambda_{1}+\mu c}
$$

where these quantities have been defined in Eqs. 16 and 17 . If one now assumes that $\mu=\sigma_{c} \vec{V}$ as suggested earlier by Connors and West, 37 then the capture cross section becomes,

$$
\sigma_{c}=\lambda_{1}\left[\Delta H /(\Delta H)_{s}\right] / \bar{V} c\left[1-\Delta H /(\Delta H)_{s}\right]
$$

In principle, to be able to calculate $\sigma$, one must measure the angular correlation for a serfes of specimens containing lenown vacancy 
concentrations ranging from amours too small to affect the distribution to quantities that totally contral it (saturation). In the case of plastic deformation this is not possible at present, but foil irradiated specinens it is in principle, because it is possible to irradiate specimens in such a manner as to create only vacancies. Combining flrediation and deformation experiments, Snead et al. ${ }^{42}$ were able to deduce the value $\sigma_{c} \cong 10 \times 10^{-16} \mathrm{~cm}^{2}$ for Fe.

The effect of vacancies alone as compared to a combination of vacancies and dislocations was demonstrated clearly for the first time by the work on iron. The hrradiation was performed at a cemperature such as to introduce only vacancies. When the sanples vere annealed at $100 \mathrm{C}$ the changes in angular correlation caused by the Irradiation disappeared (See F'ig. 9), whereas those induced by the deformation showed no recovery. Vacancies are immobile at 100C, but Snead et al. argued that the carbon known to be present in the samples had migrated to the vacancies and eliminated them as trapping centers. The deformed samples only exhibited a change following an annealing treatment at $250 \mathrm{C}$, a temperatuxe where single and divacancies are believed to be mobile. After a $400 \mathrm{C}$ anneal further changes vere observed due to the reduction of small clusters. Thus, the remaining deviation of the angular correlation distribution fron that of a well annealed state could be at ributed to dislocation along (Fig. 10). This technique of separating vacancy effects from those of othar latice defects should prove useful in future defect studies.

Connors, Crisp and West ${ }^{40}$ have analyzed in detail the temperature dependence of two-photon angular-correlation data in their paper. They illustrate the application of the trapping modsl to the determination of the vacancy-formation energy. This may prove to be one of its most useful functions. They point out that much information can be dertved from purely statistical considerations of carefully taken data. Whay maintain that changes in the angular distributions arise from 2 . 
decrease in anthilation with core-state electrons while to a first approximation the partial annihilation rate with conduction electrons remains essentially unchanged as the remperature increases. First. they normallze theit data by fitting the angular-distribution curves to a single Gausstan at large angles. This has the effect of increasing the peak heights. Next, they define a function $h(T)$ much as Snead and his colleagues have done. The function $h(T)$ is given as

$$
h(T)=\frac{\rho\left(p_{z}=0\right)}{\int \rho\left(p_{z}\right) d p_{z}} .
$$

where $p\left(p_{z}\right)$ is the measured angular distribution. If positrons anmihtlate tn normal regions of a metal to gield a normalized helght, $h_{1}$ and In a vacancy to give the corresponding height, $h_{2}$, then it can be shown from the trapping model that

$$
\ln \left(\frac{h-h_{1}}{h_{2}-h}\right)=\ln \left(a T^{1 / 2}\right)-E_{I V}^{F} / \operatorname{lgT} \text {. }
$$

Thus, Connors et a1. have plotied semi-logarithmically $\operatorname{In}\left\{\left(h-h_{1}\right) /\left(h_{2}-h\right)\right\}$ versus $1 / \mathbb{T}$ to obtain a straight line whose slope corresponds to a vacancy formation energy of $0.52 \pm 0.05 \mathrm{eV}$. The value quoted in the literature from $x$-ray lattice parameter and lengthchenge measurements is $0.44 \mathrm{eV}$. Since both types of experiments are equilibrium measurements, it is not easy to say at this point which is the more accurate. However, some speculations can be made, The surve of in(T) vS. T reveals that in reality $h_{1}$ has a temperature depencience so that it must be replaced by $h_{1}(T)$ in the equation above. A Iincar dependence has been assumed by Conncrs et al. for the function $\mathrm{h}_{1}(\mathrm{~T})$. On the other hand, $\mathrm{h}_{2}$ is assumed to be temperature sndependents. At this time one cannot compistely justify these assumptions. The observed change in the total annihilation rate is attributed to a 
decrease in core electrons around a positron in a vacancy. However, it is difficult to justify theoretically the identification of the Gaussian component of the angular distribution solely with core anninio lations. The extrapolation of this distribution to $p_{2}=0$ is also open to question. Une difficulty that the positron and comblned lattice parameter - length change experiments have in common is that at elevated tenperatures the probability of divacancy formation is not altogether negligible. The positton experiments are analyzed by ignoring this fact, whereas the lattice-parameter length-change experiments heve been interpreted wh allowances for higher-order vacanci clusters. At the moment one rould have to state that the latter method probably yields arore correct formation energies. However, if the theoretical basis for the positron experiments can be strengthened, there is every reason to belleve that they will rival other experinental methods for deducing the desired quantities.

As to which measurement, positron wean lifetime or two-photon. angular correlation, is more sensitive to the presence of defects, Delkhtyar and Cižek ${ }^{43}$ have recently argued in favor of the latter experiments. They have fnvestigated the effect of temperature upon tooquantum amihilation in deformed 4-9's nickel at 77 and $300 \mathrm{~K}$." Their aralysis consisted of deducing the parabolic part of each measured distribution and taking the ratio of this area $\left(\mathrm{s}_{\mathrm{p}}\right)$ to the total area under the curve (s). They found that $s_{p}^{300} / s_{p} 77$ i 1.35 . On the other hamd, substituting the appropriate data for $\kappa^{300}, \kappa^{77}, \tau_{1}$ and $T_{2}$ Into the expression for the mean lifetime from the trapping nodel, they Found that $\bar{\tau}^{300} / \bar{\tau}^{77}=1.05$. Thus, they concluded that the cemperature effect could not be detected readily by lifetime measurements, but could be observed easily in two photon angular correlation experiments. Of course, one can ccmbire data fron both measurements and seeis a selfconsistent solution. This has been proposed by Triftshäuser ef 0104 
We recall according to the trapping wodel that the rate of annihilation at time $t$ and temperature $T$ is:

$$
\mathbb{R}(t, T)=\frac{\left(\lambda_{1}-\lambda_{2}\right)\left(\lambda_{1}+\mu c\right)}{\lambda_{1}+\mu c-\lambda_{2}} \exp \left[\left(-\lambda_{1}-\mu c\right) t\right]+\frac{\lambda_{2} \mu c}{\lambda_{1}+\mu c-\lambda_{2}} \exp \left(-\lambda_{2} c\right)
$$

The intensitfes associated frith the two decay modes are

$$
\begin{aligned}
& I_{1}(T)=\frac{\lambda_{1}-\lambda_{2}}{\lambda_{1}+\mu c-\lambda_{2}}, \text { and } \\
& I_{2}(T)=\frac{\mu c}{\lambda_{1}+\mu c-\lambda_{2}} .
\end{aligned}
$$

Note that $I_{1}+I_{2}=1$. Thus,

$$
I_{2} / I_{1}=\mu c / \lambda_{1}-\lambda_{2}
$$

If $\mu c=\mu_{0} \exp \left(-E_{I V}^{\mathrm{F}} / k T\right), \operatorname{lnI} I_{2} / I_{1}$ vs, $T^{-1}$ should give $\mathbb{E}_{I V^{*}}^{\mathrm{F}}$.

We already know from angular-correlation analysis that if we assume the distribution to be the sum of two distributions weighted by the fraction of free and trapped positions, respectively, then we may use ang polnt on the distribution to find,

$$
\ln \left[\frac{F(T)-F_{1}}{F_{2}-F(T)}\right]=-\frac{E_{I V}^{F}}{k T}+\ln \frac{\mu_{0}}{\lambda_{n}} \text {. }
$$

The angular correlation experiment can be performed by counting in the tatl of the distribution for a long time, for example. The lifetime measurements, however, still demand the resulution of two very similar 1,ifetimes, $\lambda_{1}$ and $\lambda_{2}$. 


\section{Theory of Posteron-Vacancy Interaction}

Throughoul this review we have alluded to the need for a theoretis cal basis for understanding the behavior of positrons at defects. Very little has been published so far to satisfy this need. Hodges 45 thas attenpced to explain the trapping process in terms of an effective potential well representing the vacancy. The depths of the well, ${ }^{\prime}{ }^{D}$ is the difference between the botton of the positron energy band in the solid and the potential felt by the positron inside the vacancy. The effective positron-ion interaction $V(r)$ is calculated by treating the positron and its polarization cloud as a neutral pseudo-atom interacting with lattice atoms through a pairwise potential. Positron-electron correlations are neglected so that their influence upon the binding of positrons to vacancies is not taken into account. The positron effective potential is determined by the expression

$$
v_{t}(\vec{r})=\sum_{\overrightarrow{2} \neq 0} v(|\vec{r}-\vec{x}|)
$$

$V_{t}(\vec{r})$ has the behavior shown in Fig. 11. In the figure, the vacancy is at $r=0$, and $R_{a}$ is the radius of the Wigner-Seitz sphere surrounding. the, vacancy.

The salient features of the positron potential are:

1) Strong Coulomb repulsion near ion centers, and

2) Screening of the Cowlamb interaction in the Interstitial and vaciancy regions.

Hodges' computation consists of searching for localized bound states at energies below $v_{t}$. Thus, the problem is equivalent to that corresponding to a trapping well of depth $V_{t}$ and width $R_{a}$ Inclusion by Hodges of electron-electron correlations increases the efficiency of screening and reduces the lowest potential in tho host lattlce, $V_{W . S .}$ Hodges calculates values of $V_{t}$, the trapping pptentflal; as well as the 


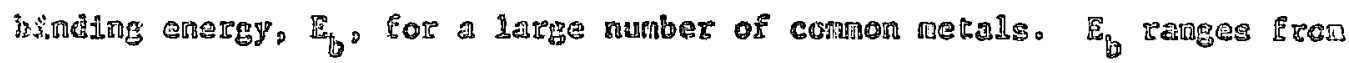

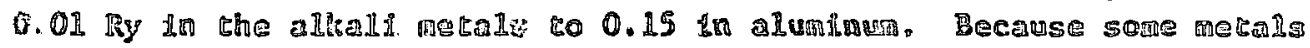

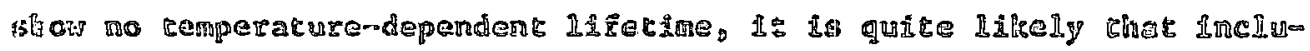

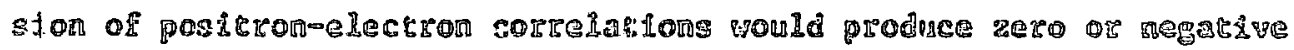
binding energies for then.

Hodges also estimated the tralpping race per unit vecancy concentra

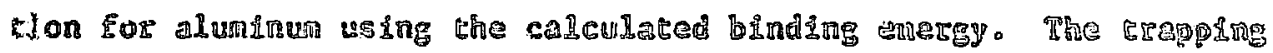
process is similar to the thermalization prosess edleulated by carbotce

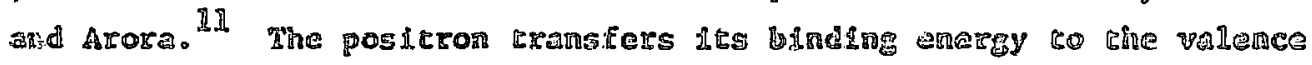

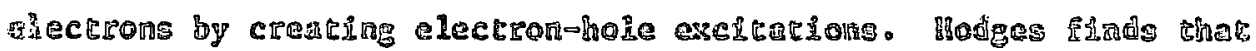

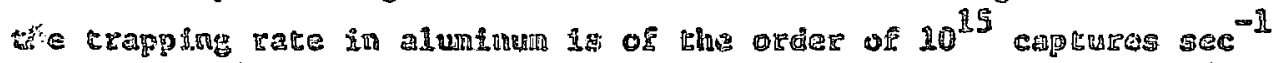
(p'ac.cone. $)^{-1}$. Thus for vecamesp concentrations greater enan $10^{-5}$ the

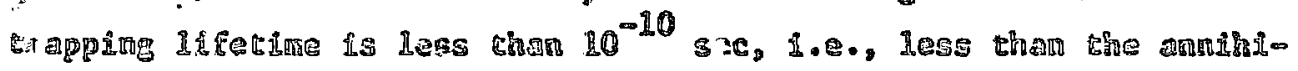

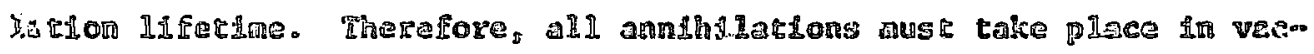

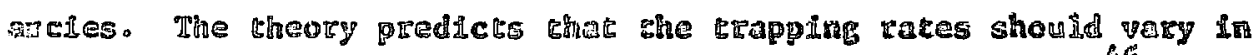
proportion to the bituling emergy. Recentiy, Kusinss et as. 46 have aif allyzed the benperature depenturence of angular correlation data for

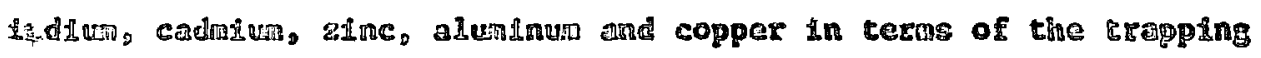
מ;

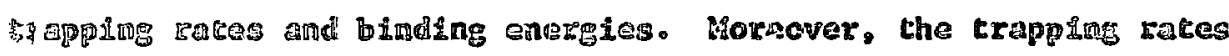

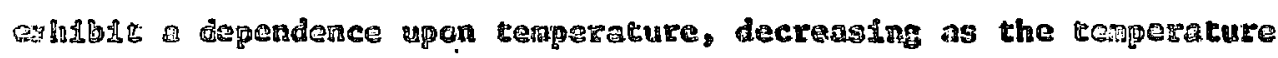
haes.

IRnSGidse et all. 16 have painted out an interesting feacure of the

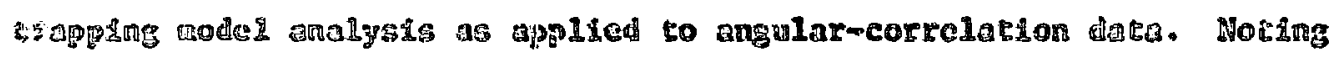

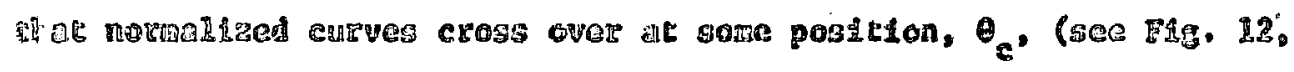

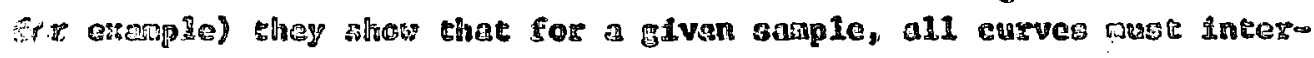
stet at the stre pasition. If they do not, there 1 s sene problen fro

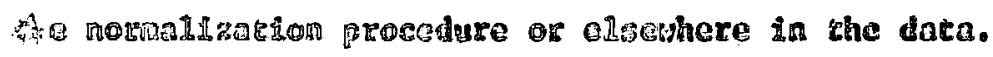




\section{SPECULARIONS AND CONCLUSIONS}

Thopefully, the foregoing review demonstrates that chere is a gretro

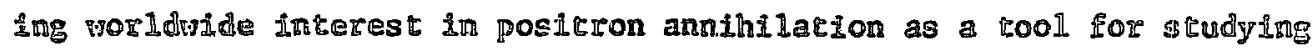
defects in metals. This conclusion was reinfurced by' the discussions and papers presented at the recent Second Incernational Conference on Positron Annihilation held at Queen's University in IRIngston, Canada. If is nos quite clear, however, that prograss in the anplication of positron amaluilation to latrice Imperfections requires further thecretical underpinnlags. For extample, there remains the question of how one ought to treat the behavior of a positron in a metal. Should it be though of classicslly as a free particle weakly interacting with the lattice, or quantrum-mechanically as an extended wave packet? In the fonter case, one carn speale of the positron capture cross suction only of trangition rates fror free to bound states. The two viewpoints have been urtirod, for example, by Brandt and Waung 47 and by Hodges, 45 respectively.

Angther aspect of the inferaction between positrons and electrons in mecals thas 50 do with the relaclve importance of annihilation with core and conduction electrons at defects. A complete theory must be able to sort out and to predict quantitative changes in these two anmihitation rates due to positron-defect interactions. This requires (4) laborledge of changes in electronic structure arising from defects as vell as a proper treatment of positron-electron interactions. At present it would appear that the pseudo-potential formalism can prove to be a powerfull tool for such theoretical investigations.

There is evidence, too, that positrons can be used t:o probe chemical inpurities in metals if the impurities possess charge states that are sufficfently different from the host lattice atoms. Equalization between the Perni level at the Impurity atom site and Its value elsewhere requires a transfer of charge. In addition, there are size-effects 
arising from the difference In atomic radif between host and impurfity atcms. These two effects produce a local perturbation in electronic structure that can be detected by the positron. Recent calculations and experiments ${ }^{48}$ on 1 ithiun-magnesium alloys appear to support these concepts. They suggest that positrons must be sensitive to very scall clusters of impurity atoms which are, in effect, precipitation nuclei.

We would be remiss if we did not point out before concluding that there exists a growing body of work dealing with positron-defect interactions in nonnetals, e.g., sodium chlordde, potassiun chloride and quart:. These Investigations iIlustrate a whole new approach to the study of color centers. They represent a complementary technique that should become increasingly important in the effort to understand defects in lonic crystals.

Thus, we conclude that the potential for useful research on the defect solid state which is presented by the phenomenon of positron annihilation will be realized and fully exploited in the next few years.

\section{REFERENCES}

1. S. DeBenedett1, R.E. Be11 and M. Deutsch, in Beta- and Gamma-Ray Spectroscopy. R. Siegbahn, Editor, p. 672, Interscience Publishers Inc., New York, 1955.

2. R. Ferre11, Rev. Mod. Phys. 28, 308 (1956).

3. P.R. Wallace, Solid State Physics, F. Seitz and D. Turnbull, Editors, Vol. 10, p. 1, Acadenic Press, New York (1960).

4. A.T. Stevart, Proceedings of the Internations Conference on Positron Annihilation, A.T. Stewart and L.0. Roe11:g, Editors, p. 17, Academic Press, New York, 1967.

5. M. Deutsch and S. Berko, In Alpha-, Beta- and Gama-Ray SpeccroScopy, K. Siegbahn, Editor, p. 1583, VoI. II, North Holland Publishing Co., Ansterdam, 1965. 
6. Cor. Majumdar, in theory of Condensed Matcer, P. 829. Intermational Atomic Energy Agency, Vienna, 1968.

7. K. Rama Reddy and R.A. Carrigan, Jr., Nuovn Cim. 66n, 105 (1920)。

8. H.W. Soudex, Ph.D. Thesis 1969, Iowa State University, Ames, Iorge (unpublished).

9. H.L. Weisberg, Ph.D. Thesis 1965, Brandelis University, Walther, Massachusetts (unpublished).

10. G.E. Lee-Whiting, Phys. Rev. 97, 1157 (1955).

11. J.P. Carbotte and H. Arora, Can. J. Phys. 45, 387 (1967)。

12. H.J. Mikeska, Zeic. f. Phys. 232, 159 (1970).

13. J.P. Carbotte, Phys. Rev. 155, 197 (1967).

14. B. Bergersen, in Soft X-Ray Band Spectra, D.J. Fablen, Fistor, Academic Rress, London, 1968.

15. A. Själander and M.J. Stott, Sol. State Comm. $\underline{8}$, i881 (1970).

16. A. Held and S. Kahana, Can。 I. Phys. 42, 1908 (1964)。

17. S. debenedetts, C. Corram, Ho. Konneker, and I. Primakoff, Phys. Rev. 77, 208 (1950).

18. S. Berko and J.S. Plaslecte, Phys. Rev. 112, 1877 (1958).

19. A.C. Damask and G. J. Dienes, in Point Defects in Mielalls, Gordon and Breach Science Publishers, Inc., New York 1964.

20. S. Antelinckx, in Solid State lyhsics, F. Seitz and D. Turnbull, Editors, Supplement 6, Academic Press, New York, 1964.

21. Vacancies and Interstitials in Metals, A. Seegex, Do Schumacher, W. Schllling and J. Diehl, Edittors, North Holland, Amstexdam, 1970.

22. I. Ya. Dekhtyax, Czech. I. Yhys. 1.2B, 1503 (1968).

23. I. Ya. Dekhtyar, D. A. Levina, and V.S. Mikhalenkov, Sov. Pliys.* Dok1. 9, 492 (1964).

24. S. Berko and J.C. Exskine, Phys. Rev. 19, 307 (1967).

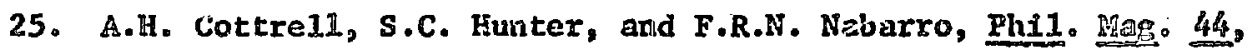
1064 (1953). 


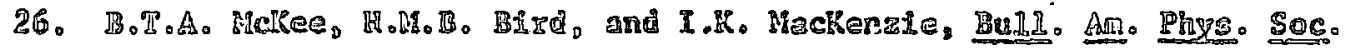
12. 689 (1967)。

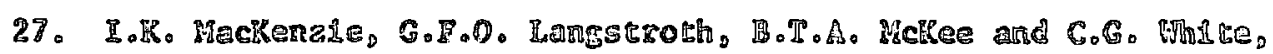
Cam. I. Phys. 42, 1837 (2064).

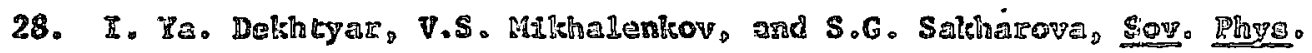
Solid State 11, 2647 (1980).

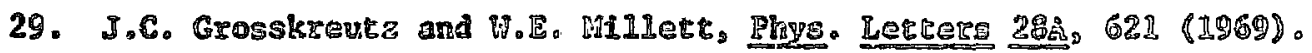

30. I.x. Makkenzlie, Phys. zerters 30A, 115 (1969).

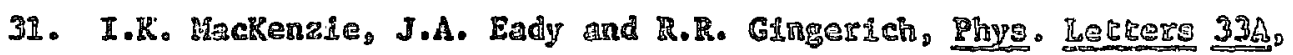
279 (1970).

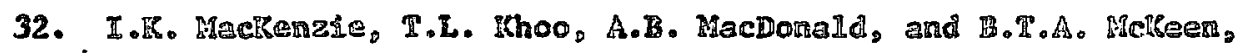
Phys. Bev. Letcers 19,946 (1967).

33. ㄱ. Heisberg and S. Berko, Phys. Rev, 154, 249 (1967)。

34. Wr. Brandt and H.F. Maung, Phys. Letters 27A, 700 (1968).

35. H. Brandl, In Proceediags of the International Conference on Posteron Aminilation, A.I. Stewart and L.0. Roellig, Rditors, D. 280, Academic Press, Nev York 1967.

36. B. Bergersen and M.J. Stote, Sol. State Comm. I, 1203 (1969).

3\%. D.C. Connors and R.N. West, Phys. Letters 30A, 24 (1969).

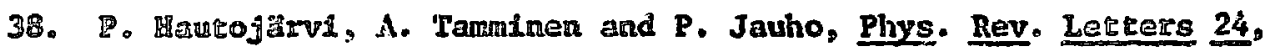
459 (1970).

39. D.C. Connors, V.H.C. Crisp and R.N. West, Phys. Lettere 33A, 180 (1970).

40. D.C. Connors, V.H.C. Crisp and R.R. West, I. Phys. E.: Metal Phys. I. 355 (1971).

4I. J.IF. Kusmiss, C.D. Esseltine, C.L. Snead, Jr., and A.B. Golland, Phys. Letters 32A, 175 (1970).

42. C.L. Snead, Jr., A.N. Goland, J.H. Kusmiss, H.C. Huang aถd R. Meade, phys. Rev. 3B, 275 (1971). 
43. I. Ya. Dellicyar and A. Cizek, Phys. Letters 34A, 345 (1971).

4h. H. Trifteshuser, B.I.A. Mclee and A.T. Stewart, BuIl: Ario. Phys. Soe. 15, 812 (1970).

45. C. H. Hodges, Pligs. Rev. Lecrers 25, 284 (1970)。

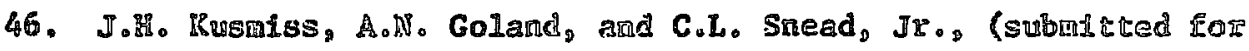
publicacion).

47. W. Brandt and H.F. Haung, Rhys. Rev. M15 3, 3432 (1971)。

48. P. Kubiea, B.T.A. Mickes, A.T.Stewart and H.J. Stott (to be publisted). 


\section{FIGURE CAPTIONS}

Fig. I. The static pajir correlation function according to Sjölander and Stotr (full line) and using linear response theory (broken line). (Ref, 15).

Fig. 2. Schematic diagram of a typical 2-photon angular-correlation experiment.

Fig. 3. Angular distribution for aluminum. The points are experimental data for the [111] drection in aluminum, and the solid curve is a theoretical one normalized to the expertmental counting rate at $0^{\circ}$. (Ref, 18).

Fig. 4. A slice through the Fermi surface for a free-eiectron gas showing how the parabolic angular distribution arises.

Fig. 5. Comparison of pair monentum distribution spectrum from Doppler-broadening technique and angular-correlation technique; points $(0)$ and $(\theta)$ represent two methods of analysis of Doppler-broadening data; $(\Delta)$ are angular-correlation data from A. T. Stewart, Can. Jour. Phys. 35, 168 (1957). (Ref. 7).

Fig. 6. Block diagram of slow-fast delayed coincidence method for time spectroscopy of annihilation photons.

Fig. 7. Typical appearance of prompt and delayed spectra obtained by the delayed coincidence technique depicted in Fig. 6.

Flg. 8. The correlation between hardness and positron erapping in copper of high purity. (Ref, 31). 
Flg. A. Angular correlation curves comparing irradiated itron raleh iron irradiated and then annealed at $100^{\circ} \mathrm{C}$ for $1 \mathrm{ho}$. The dashed curve depicts the angular correlation for the annealed unirradiaced specimen. (Ref. 42).

Fig. 10. The effects of deformation and annealing on angurlat cois relation in lron. (a) compares 1 ron piastically deforamed to $16 \%$ with an undeformed anealed spectnen and (b) corj: pares the same deformed sample before and after annealing at $250^{\circ} \mathrm{C}$ for $1 \mathrm{~h}$. (Ref. 42)。

Fig. 11. The potential $v_{t}$ (solld line), formed by the superposition of repulsive positron-1on interactions (dashed lines). ploticed against distance $r$ along a line of atomic centers from the vacancy center in Al. The horizontal dotted litre represents the bottom of the positron energy band, and $v_{e^{3}}$ the equivalent trapping potential, is determined by the zero-point energy of the positron $v_{2 p}$, and the lowest potential in the host lattice, $\mathrm{v}_{\mathrm{ws}}{ }^{\circ}$ (Ref. 45).

Fig. 12. Angularmcorrelation results for In at 298K and 403R. Both surves are normalized to the same total area. The shaded portion (marked $\Delta A$ ) is used in deducing the fraction of positrons which annihilate from traps. (Ref. 46). 


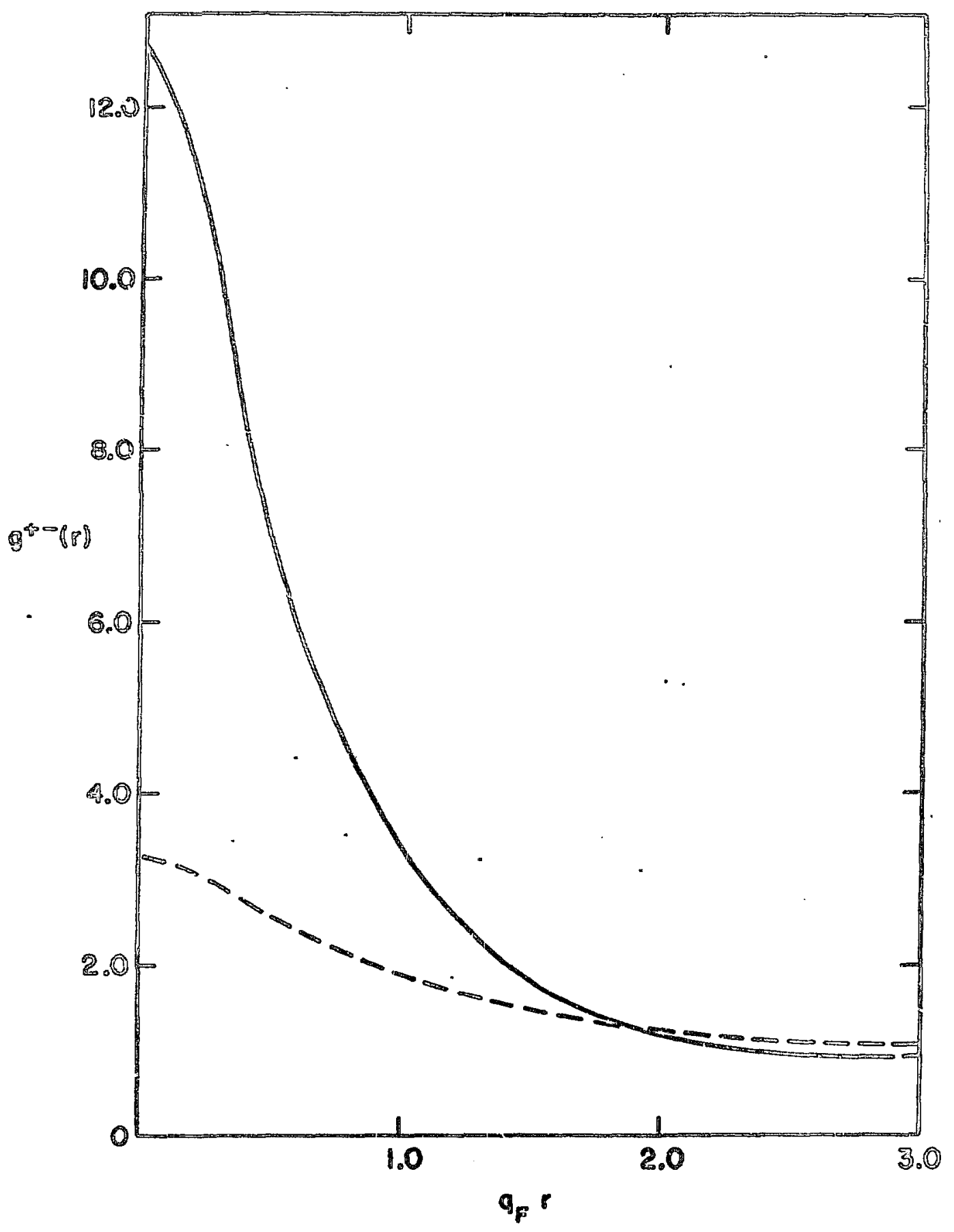

[586.8 


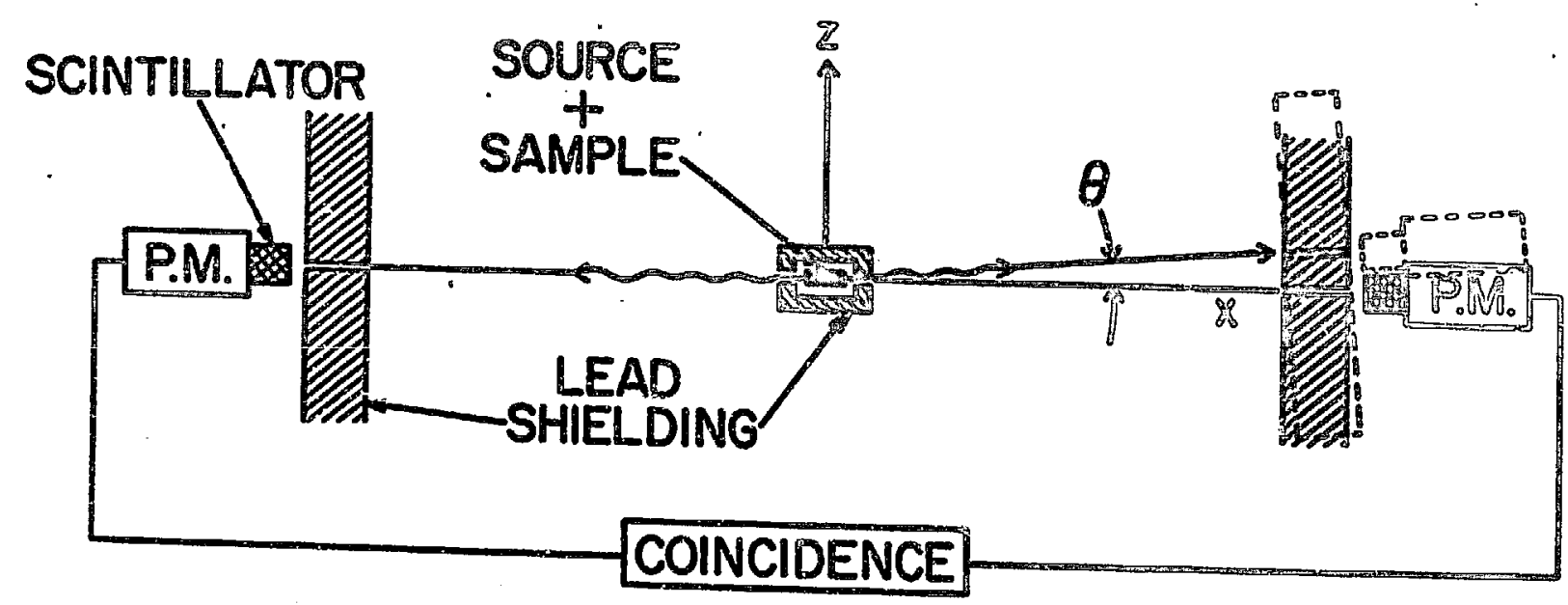




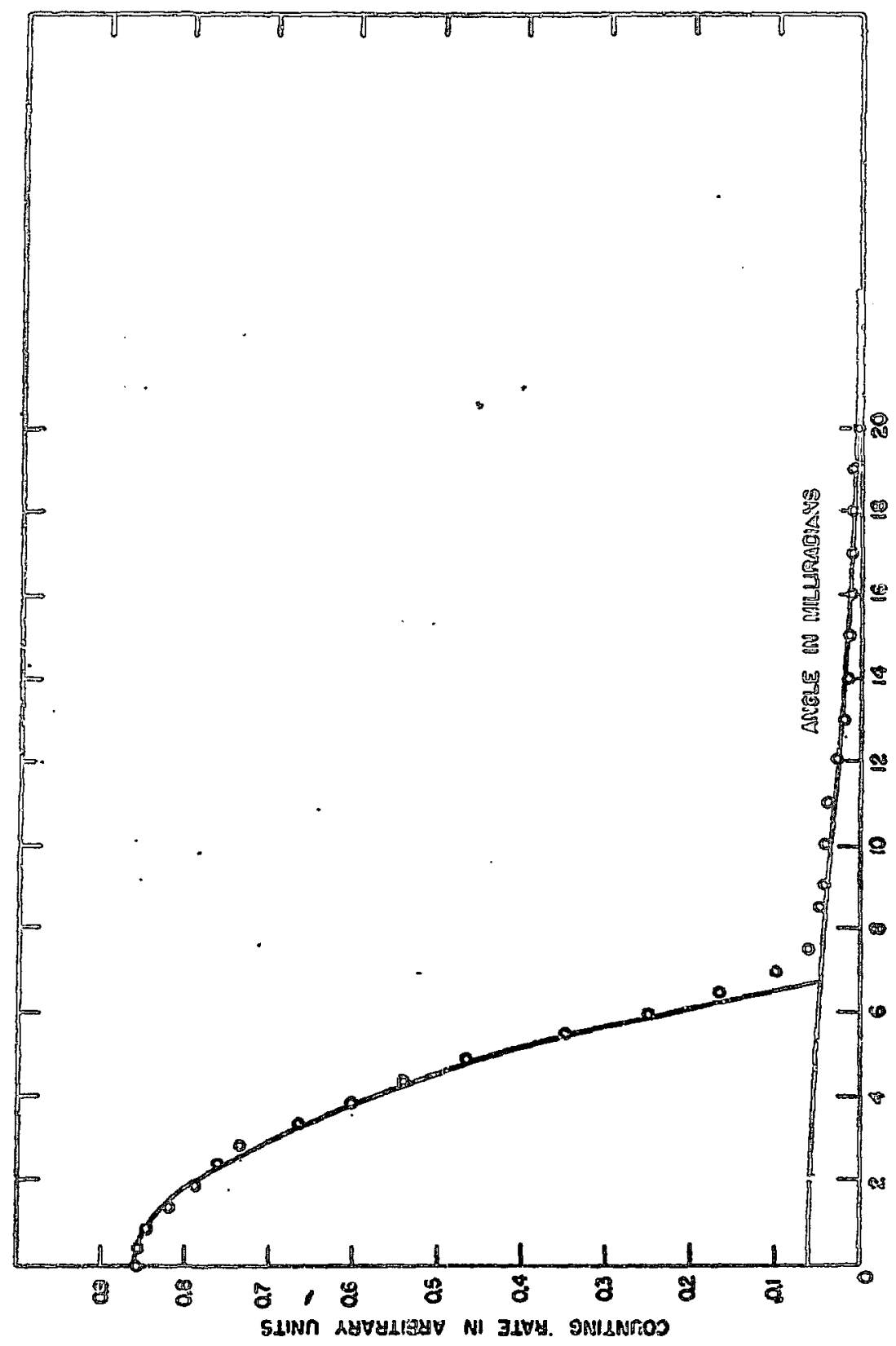

Ple. 3 


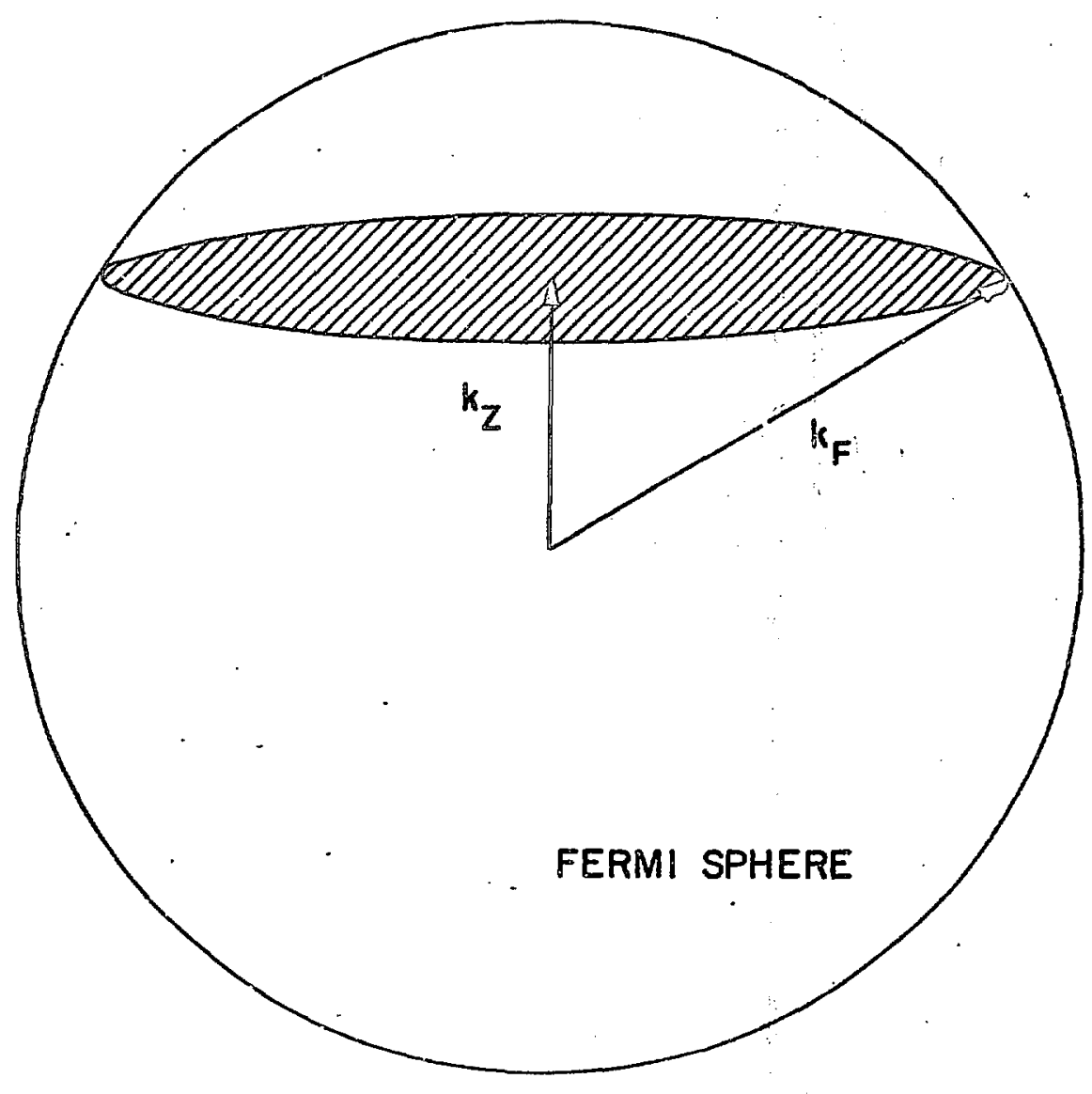

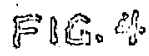




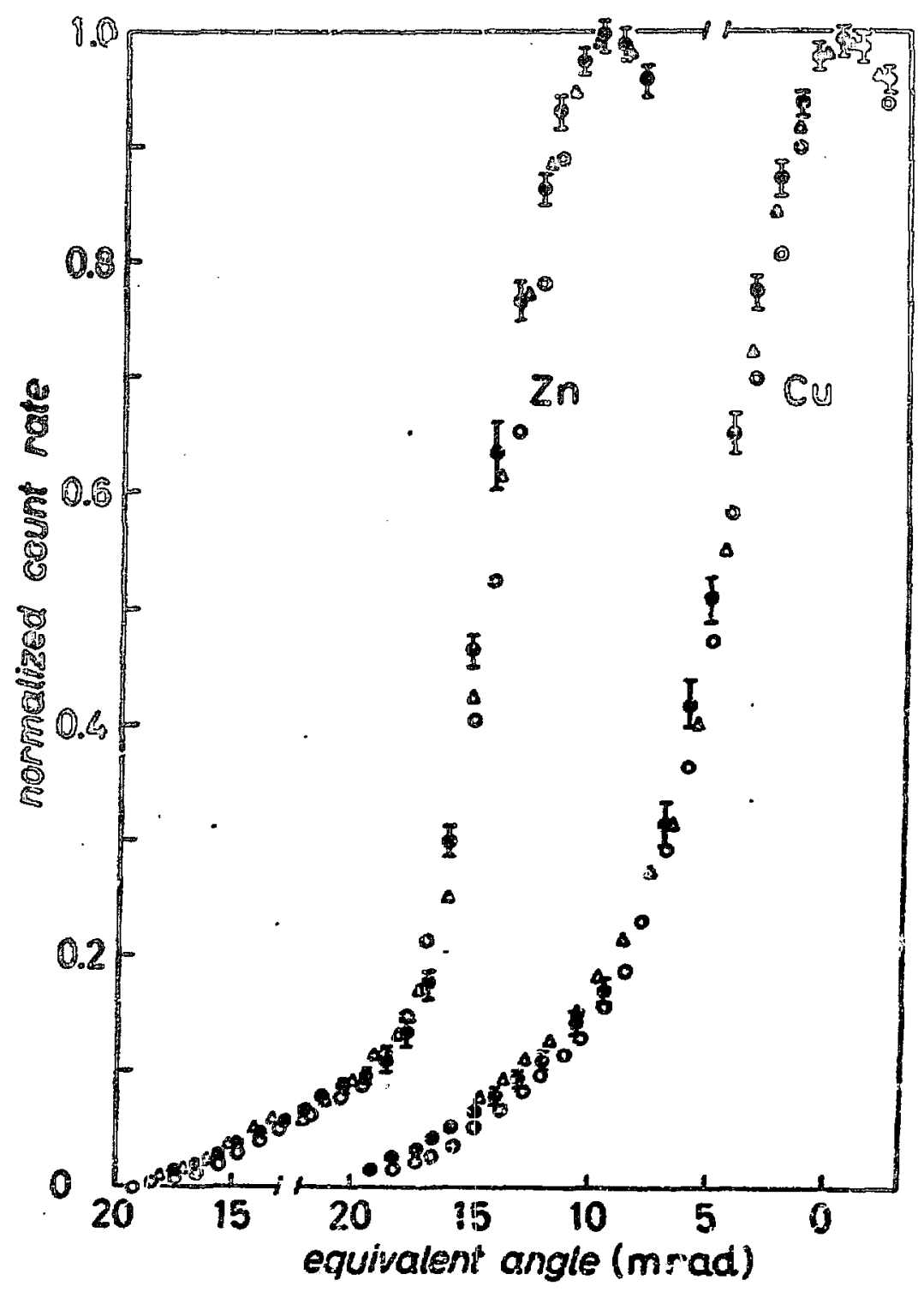

P0.5 


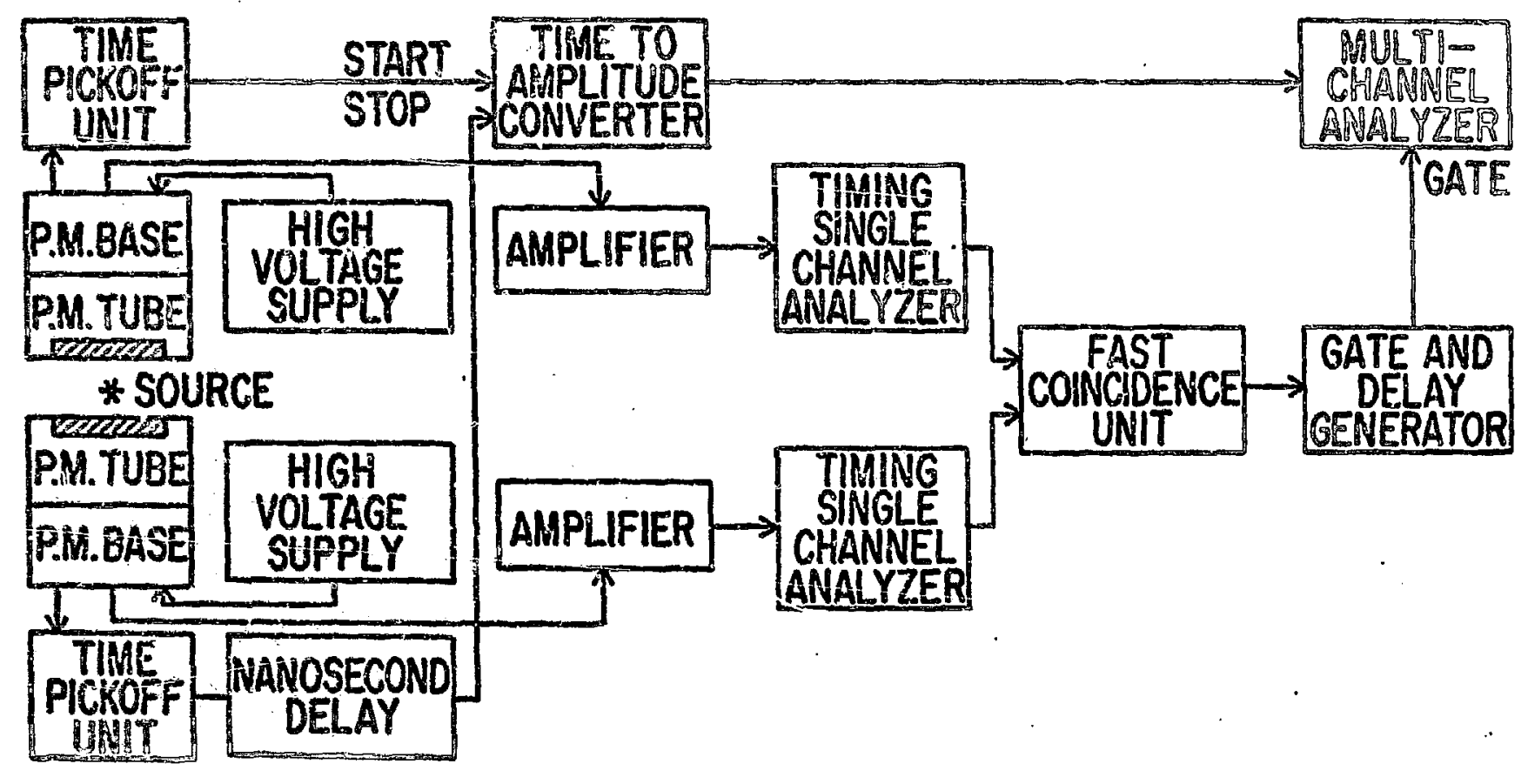




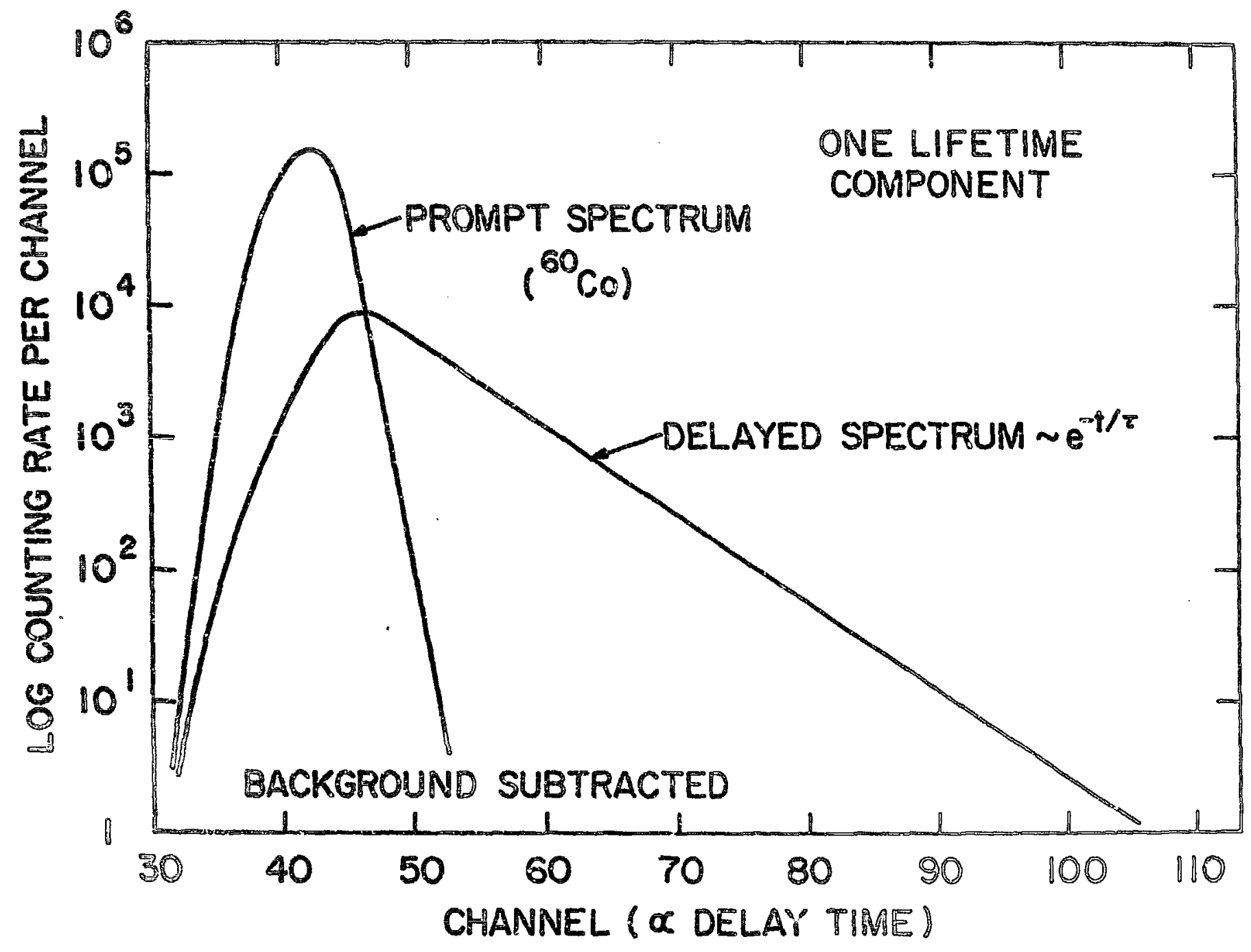




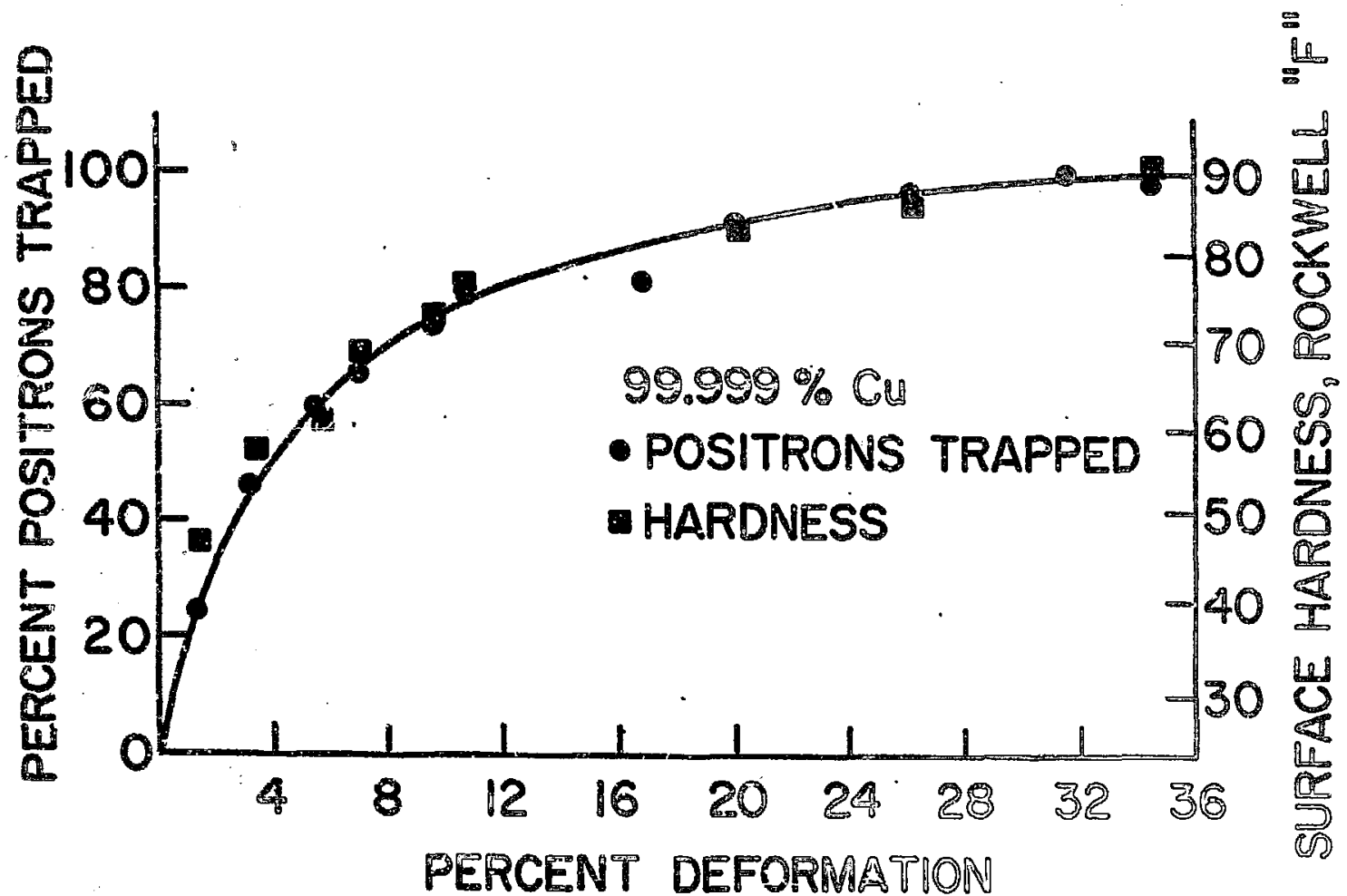




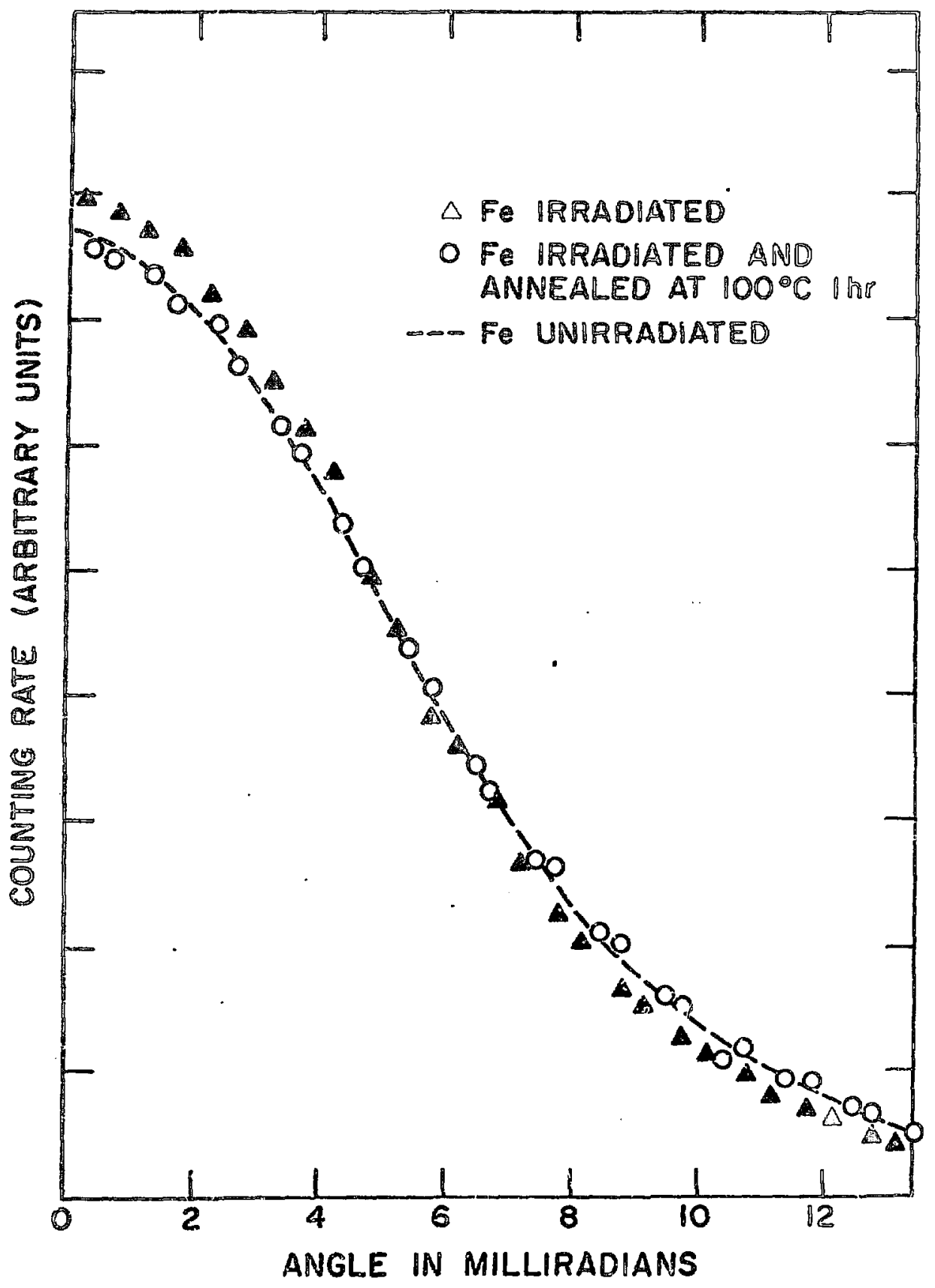

5169 
COUNTING RATE (ARBITRARY UNITS)
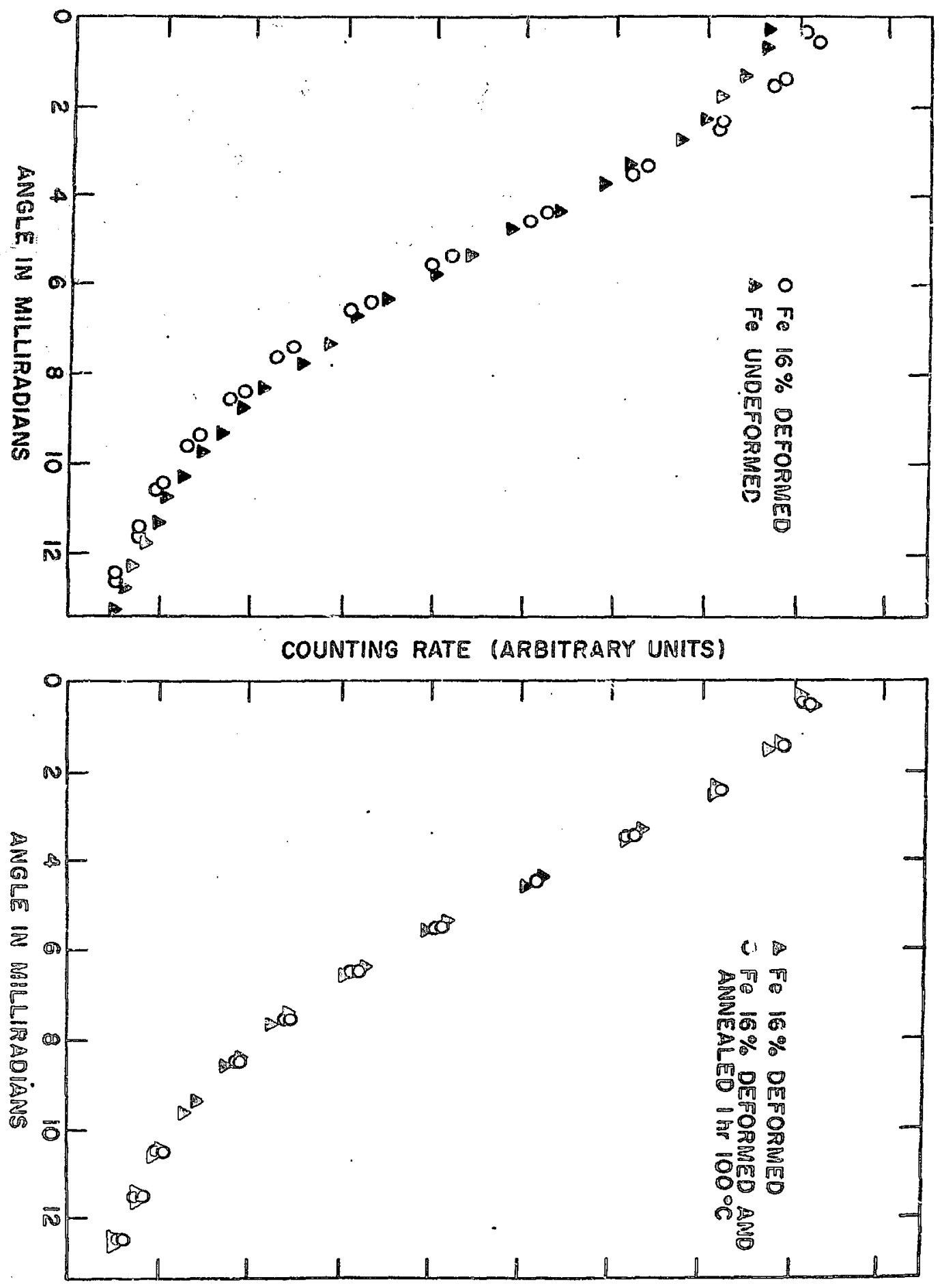


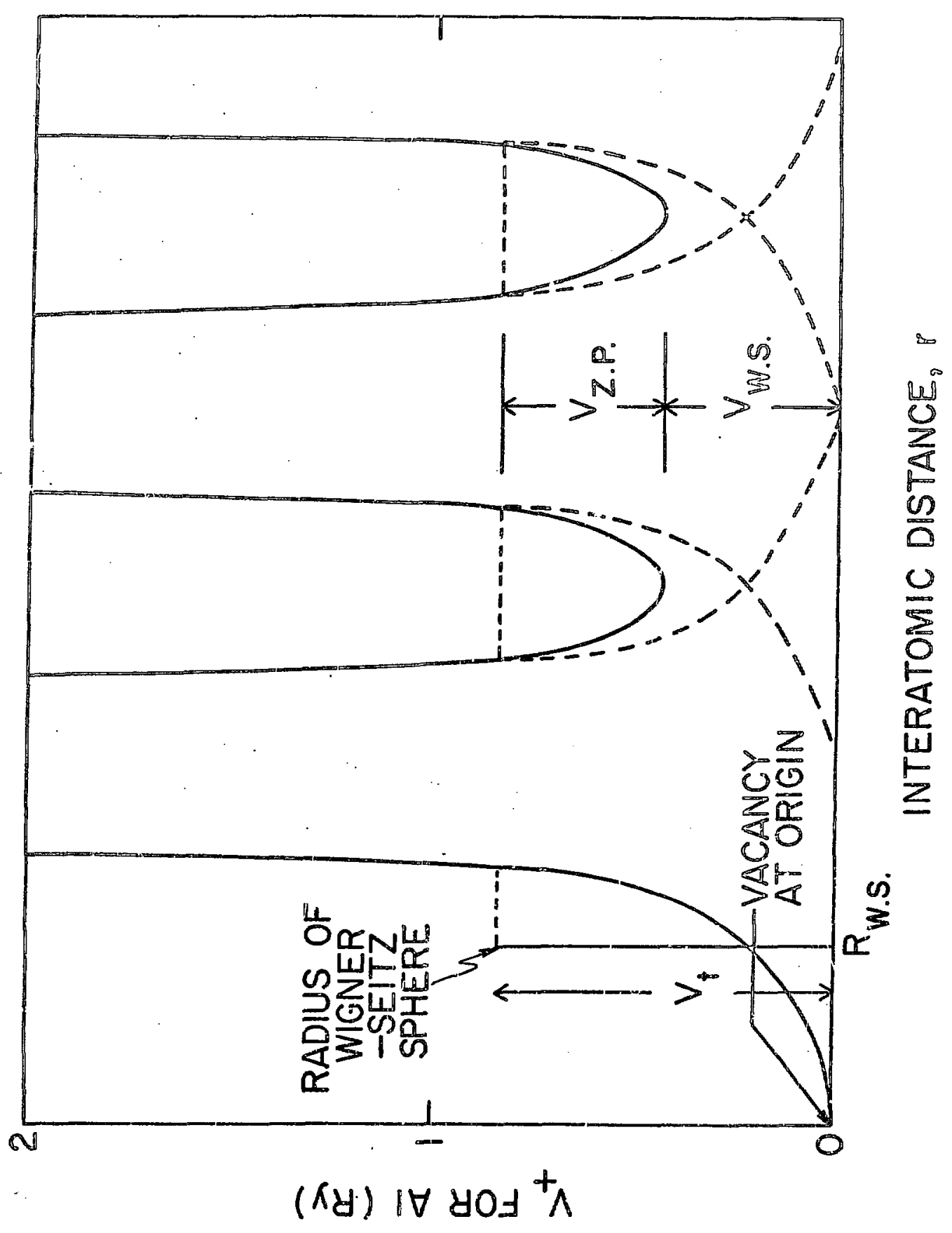

FPA. 


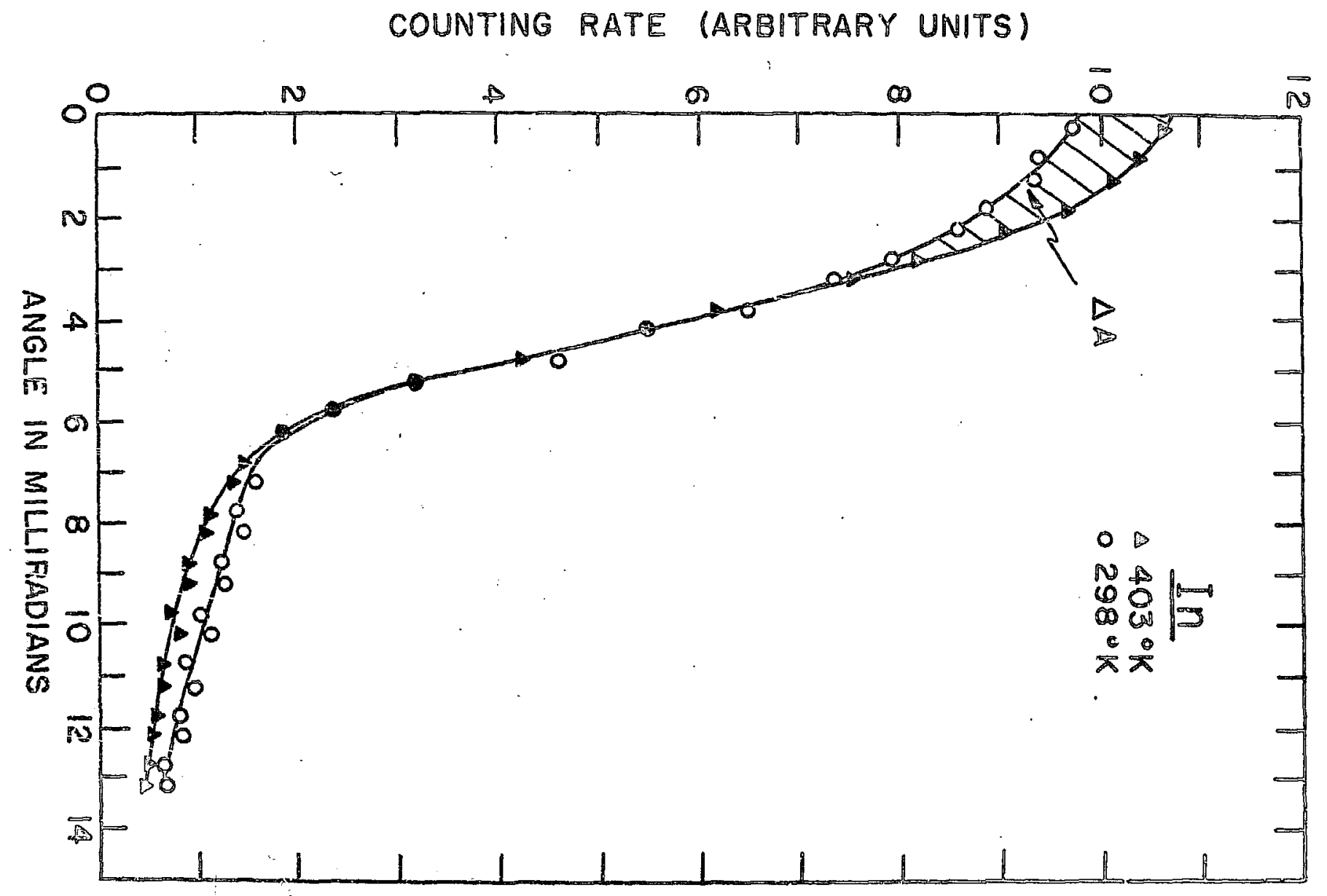

